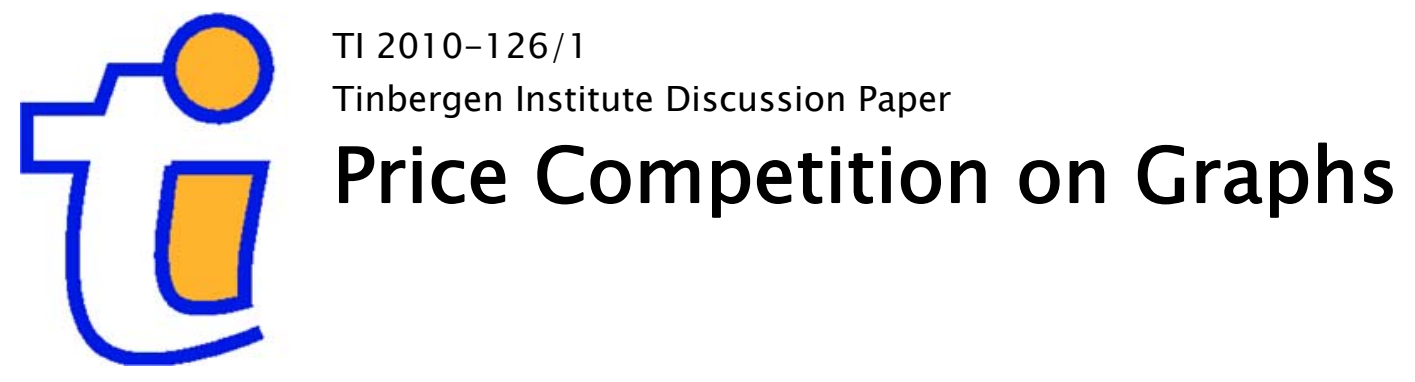

Adriaan Soetevent

ASE, University of Amsterdam, and Tinbergen Institute. 
Tinbergen Institute is the graduate school and research institute in economics of Erasmus University Rotterdam, the University of Amsterdam and VU University Amsterdam.

More TI discussion papers can be downloaded at http://www.tinbergen.nl

Tinbergen Institute has two locations:

Tinbergen Institute Amsterdam

Gustav Mahlerplein 117

1082 MS Amsterdam

The Netherlands

Tel.: +31(0)205251600

Tinbergen Institute Rotterdam

Burg. Oudlaan 50

3062 PA Rotterdam

The Netherlands

Tel.: +31(0)10 4088900

Fax: $+31(0) 104089031$

Duisenberg school of finance is a collaboration of the Dutch financial sector and universities, with the ambition to support innovative research and offer top quality academic education in core areas of finance.

DSF research papers can be downloaded at: http://www.dsf.nl/

Duisenberg school of finance

Gustav Mahlerplein 117

1082 MS Amsterdam

The Netherlands

Tel.: +31(0)20 5258579 


\title{
Price competition on graphs
}

\author{
Adriaan R. Soetevent* \\ University of Amsterdam (ASE) and Tinbergen Institute
}

August 11, 2011

\begin{abstract}
This paper extends Hotelling's model of price competition with quadratic transportation costs from a line to graphs. I propose an algorithm to calculate firm-level demand for any given graph, conditional on prices and firm locations. These graph models of price competition may lead to spatial discontinuities in firm-level demand. I show that the existence result of D'Aspremont et al. (1979) does not extend to simple star graphs and I conjecture that this non-existence result holds more generally for all graph models with two or more firms that cannot be reduced to a line or circle.
\end{abstract}

JEL classification: D43, L10, R12

Keywords: spatial competition, Hotelling, graphs.

\section{Introduction}

Firms face two opposing incentives in the decision where to locate relative to competitors. A location close to one's competitors maximizes the opportunities to capture one's competitors' consumers, but at the same time, little spatial or product differentiation increases price competition among firms. Hotelling (1929) introduced a stylized linear model of spatial (product) differentiation to analyze which of these is the dominant force ${ }^{1}$ The current paper generalizes Hotelling's line model of spatial (product) differentiation to graphs. For markets with two

*Universiteit van Amsterdam, Amsterdam School of Economics/AE/IO, Roetersstraat 11, 1018 WB Amsterdam, The Netherlands, Ph: +31 - (0) 20 - 52573 51; a.r.soetevent@uva.nl. Support by the Netherlands Organization for Scientific Research under grant 457-07-010 is gratefully acknowledged. We The study benefited from comments by Robert Adams, Nicholas Economides, Pim Heijnen, Jeroen Hinloopen, Stephen Martin and participants at the IIOC 2011.

${ }^{1}$ D'Aspremont et al. (1979) show the invalidity of Hotelling's original claim that with transportation cost linear with respect to distance, firms tend to minimally differentiate. They demonstrate that, in a model with transportation cost quadratic in distance, a price equilibrium solution exists for any pair of locations and firms maximally differentiate. Irmen and Thisse (1998) conclude that, despite differences in modeling assumptions, the outcome of most theoretical models is that firms seek to differentiate in order to avoid price competition. They 
competing firms and transportation cost quadratic in distance, I present an algorithm that calculates firm-specific demand as a function of the firms' prices and conditional on their position in the graph. In other words, for any structure of lines and intersections that one can draw on a piece of paper and the position of the firms on this structure, the algorithm will give firm-level demand. The constructed graphs may be as arbitrary as the patterns of released sticks in the game of Pick-up sticks, after the isolated sticks have been removed. Consumers are assumed to be uniformly distributed on the graph's edges. The line model with quadratic transportation cost as studied by D'Aspremont et al. (1979) arises as a special case.

The prime motivation for this extension is that in reality, firms cannot locate just anywhere on a plane but are constrained by zoning, geography and roads. As a result, observations of clustering by firms in physical space are not the exclusive result from firm conduct but may as well reflect the structure of the product space. Recent empirical studies acknowledge this and use techniques from spatial statistics to develop measures of spatial clustering that correct for this (Picone et al., 2009) $2^{2}$ To the best of my knowledge, no theoretical models exist that evaluate what price profit-maximizing firms would choose on a graph, conditional on their own location and those of competitors. Throughout this paper, firm's location will be taken exogenous. That is, I focus on the second stage of the two-stage game with firms competing in prices in the second stage after having chosen their location in the first stage.

In Section 2, I develop general notation to analyze this type of problem. It is unavoidable that the notation is somewhat unwieldy because one needs to keep track of the shortest path from each consumer to each of the firms. I will illustrate with simple examples every concept that is introduced. Section 3 presents for a market with two competing firms a formal algorithm that calculates firm-specific demand as a function of posted prices, given the firms position in the graph for a market with two competing firms. Implementation of this algorithm into software code allows one to numerically evaluate for any given graph and firm position a firm's best-response to the price charged by the other firm $3^{3}$

Importantly, a number of standard results do not carry over from the unit interval to graph

however show that when the analysis is extended from one-dimensional to multi-dimensional characteristics space while upholding the quadratic transportation costs, firms only maximally differentiate in a single dimension and thus Hotelling was "almost right".

${ }^{2}$ The presented graphs are best interpreted as models of differentiation in physical space but interpretations as models of differentiation in product space may be possible.

${ }^{3}$ The Matlab code together with documentation is available at the project's web site, www.tinyurl.com/ pcgraphs. The main program PCGraphs.m calculates for any given graph and firm locations in a number of iterations the best price-response of one firm given the price charged by its competitor. 
models of price competition. First, when transportation costs are quadratic (as I will assume throughout), spatial discontinuities in firm-level demand may occur. That is, consumer's with a preference for firm $i$ 's product are surrounded by consumers with a preference for its competitor's product. Second, in contrast to D'Aspremont et al. (1979), the assumption of quadratic transportation cost no longer is a sufficient condition for the existence of a Nash equilibrium in pure strategies 4

In Section 4, two theorems are derived on the existence of pure- and mixed-strategy price equilibria. The first theorem says that for arguably the simplest extension of the line model, the "Hotelling line with a junction" (a $K_{1,3}$ graph), there always exist firm location configurations for which the price competition game does not possess a noncooperative equilibrium in pure strategies. I compare this result with a the non-existence result in Varian (1980) and argue that the market context and line of proof is different here. This non-existence result is the main reason for not endogeneizing firm locations ${ }^{5}$ I will conjecture that for every graph with at least one node with degree 3, firms can always be located such that no equilibrium price solution exists. The second theorem states that every two-firm graph model of price competition has a mixed-strategy equilibrium for any configuration of firm locations. The latter theorem is a straightforward extension of Dasgupta and Maskin (1986b, Theorem 3) who have proven this for the line model.

The paper is related to studies on pricing on networks that have appeared, like Bloch and Querou (2009). These studies however locate firms and consumers at nodes, following the modeling methodology common in social network analysis. In particular, the edges in these models are "void": they are not inhabited by a density of consumers but only serve the purpose of connecting two nodes. The graphs presented here are fundamentally different because consumers are assumed uniformly distributed along the nodes and edges of the graph 6 This is in the spirit of Hotelling's line model, Salop's (1979) circular model and Von Ungern-Sternberg's (1991)

\footnotetext{
${ }^{4}$ The assumption of quadratic transportation cost, where disutility rises more than proportional with distance is often thought to be more appropriate in models where "distance" is not interpreted as a physical distance but proxies for the difference between the characteristics of the product bought and the most preferred variety. Within the current model, non-linear transportation costs may however reflect increased search cost: the greater the distance between the consumer and the firm, at the more crossroads the consumer has to take the right turn to reach the firm.

${ }^{5}$ See Osborne and Pitchik (1987) for the complexity of characterizing the mixed strategy equilibria even in the original Hotelling model with travel cost proportional to distance. Implementing the first-stage is also computationally difficult because in each step one has to evaluate the profits associated with infinite number of possible locations for firm $i$, conditional on the position of its competitors.

${ }^{6}$ Commuting behavior of consumers is not considered, see Claycombe and Mahan (1991); Raith (1996) for theoretical contributions and Houde (forthcoming) for a state-of-the-art empirical study.
} 
pyramid model. As a consequence, I need to introduce a considerable amount of new notation.

A number of papers (Mills and Lav, 1964; Eaton and Lipsey, 1976; Greenhut, Hwang and Ohta, 1976; Holahan and Schuler, 1981) have studied location choice and price competition on two-dimensional spatial markets with constant transport cost per unit distance and free entry. The starting point of this literature is the well-known result first asserted by Lösch (1954) and formally proven by Bollobás and Stern (1972) that, conditional on every consumer in the plane being served and constant transport cost per unit distance, a division of market demand into hexagons is socially optimal. Subsequent contributions have questioned whether the hexagonal configuration is the unique equilibrium when the number of firms is given and the extent to which this configuration results under free entry $]^{7}$ These studies have in common that consumers are assumed uniformly distributed over a plane. Instead, the current paper is concerned with studying the generic properties of spatial models of product competition with consumers uniformly distributed along the edges of a given graph. I do not study which firm configurations result under entry for any particular (class of) graphs.

I limit attention to the situation with two firms and quadratic transportation cost but conceptually, the analytical approach can be extended in a straightforward manner to cover situations with non-quadratic cost and multi-firm competition. In fact, as in the pyramid model in Von Ungern-Sternberg (1991), graphs/network structures easily allow for multi-firm competition. The difference with the model by Von Ungern-Sternberg is that no analytical solutions are available for less stylized graphs.

\section{Model and notation}

Consider a static noncooperative two-stage game with $n$ single-store firms located on a graph. Consumers are uniformly distributed on the graph. In the first stage, firms choose a location on the graph, in the second stage each firms chooses price given location.

Let $V$ denote the set of vertices (or nodes) and $E$ the set of edges. $G=(V, E)$ is a connected graph and points in $G$ are denoted by $\omega \in G$, Points $\omega$ are two-dimensional vectors. The set of all points is denoted as $\Omega=\{\omega: \omega \in G\}$. Let $d: \Omega \times \Omega \rightarrow \Re^{+}$represent a real valued weight function representing the distance between two points $\omega$ and $\omega^{\prime}$ in $G$. Individual edges are denoted as $e_{u, v}=(u, v)$ with $u \in V, v \in V$ with $u$ and $v$ the endvertices of the edge. Since

\footnotetext{
${ }^{7}$ Eaton and Lipsey (1976) demonstrate that without entry and/or exit, next to the hexagonal configuration, equilibrium configurations of squares and rectangles can occur but that the rectangular lattice seems most robust to entry.
} 
the graph is undirected, the edges are unordered: $e_{u, v}=e_{v, u}$; in what follows we take $u<v$. An edge represents the set of all points $\omega \in G$ between $u$ and $v$, that is,

$$
e_{u, v}=\{\omega \in G: \omega=\lambda u+(1-\lambda) v ; \text { for } \lambda \in[0,1]\} .
$$

In this formula, we use the two-dimensional vector representation of $u$ and $v$. The function $d\left(e_{u, v}\right)$ gives the length of edge $e_{u, v}$. A path $P_{0, k}$ is a sequence $e_{0,1} v_{1} \ldots e_{k-1, k}$ with no repeated vertex.

Firms and consumers can locate not only at vertices but also at any point of an edge. This is a notable difference with other graph-theoretic applications in economics which necessitates considerable extra notation. Firm $i$ is denoted as $f_{i}, i=1,2$. If $f_{i}$ is located at point $\omega \in e_{u, v}$, we refer to $e_{u, v}$ as the home edge of $f_{i}$ and denote it as $e^{f_{i}}$. If two firms are located at the same edge, $e^{f_{i}}=e^{f_{j}}$.

Distances Determine the distance $d_{v, f_{i}}$ of each vertex $v \in V$ to each of the firms $f_{i}$ and let

$$
C_{f_{i}}=\left\{v \in V \mid d_{v, f_{i}}=\min _{j} d_{v, f_{j}}\right\} \text { for all } i \in\{1,2, \ldots, n\}
$$

That is, $C_{f_{i}}$ is the set of vertices which are at least as close to firm $i$ than to any of the other firms. Note that $C_{f_{i}} \cap C_{f_{j}}$ is not necessarily empty, that is, vertices may belong to more than

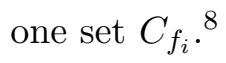

Shortest Paths Denote the different shortest paths (numbered $m=1,2, \ldots M$ ) between any two locations $\omega, \omega^{\prime} \in G$ as $P_{\omega, \omega^{\prime}}^{S_{m}}$. For simplicity, we do not index $P^{S}$ with $m$ when the shortest path between two points is unique, i.e. $M=1$. The shortest path $P_{\omega, \omega^{\prime}}^{S}$ is of the form $\omega\left(\omega-v_{0}\right) e_{0,1} v_{1} \ldots e_{k-1, k}\left(v_{k}-\omega^{\prime}\right) \omega^{\prime}$, where $\omega-v_{0}$ denotes the line segment of the home edge that connects $\omega$ with the first vertex in $P_{\omega, \omega^{\prime}}^{S}$. The difference between edges and line segments is that edges always connect two vertices whereas line segments can connect any two points $\omega$ and $\omega^{\prime}$ conditional on $\omega, \omega^{\prime} \in e_{u, v}$ for $u, v \in V$. Line segments are thus always a subset of a particular edge with its adjacent vertices. We write:

$$
e_{\omega^{\prime}, \omega^{\prime \prime}}=\left\{\omega \in G: \omega=\lambda \omega^{\prime}+(1-\lambda) \omega^{\prime \prime} ; \lambda \in[0,1]\right\},
$$

\footnotetext{
${ }^{8}$ Note that this set in general does not coincide with the set of vertices served by a particular firm.
} 
with $e_{\omega^{\prime}, \omega^{\prime \prime}}$ being an edge when $\omega^{\prime}$ and $\omega^{\prime \prime}$ are both vertices and a line segment otherwise. Let $V\left(P_{\omega, \omega^{\prime}}^{S}\right)$ be the set of all vertices included in $P_{\omega, \omega^{\prime}}^{S}$, except possibly $\omega$ and $\omega^{\prime}$ themselves if they happen to be a vertex. We will denote the shortest path distance between $\omega$ and $\omega^{\prime}$ with $d\left(P_{\omega, \omega^{\prime}}^{S}\right) . v \in C_{f_{i}}$ if and only if $P_{v, f_{i}}^{S} \leq P_{v, f_{j}}^{S}$ for all $j$.

\subsection{Change Points}

The function $V^{1}\left(P_{\omega, \omega^{\prime}}^{S}, V\right)$ gives the vertex in the set $V$ that is encountered first (excluding $\omega$ itself in case $\omega$ is a vertex) when moving along the shortest path from $\omega$ to $\omega^{\prime}$ for any $\omega, \omega^{\prime} \in \Omega$. In particular, $V^{1}\left(P_{\omega, f_{i}}^{S}, V\right)$ gives the endpoint of the home edge of $\omega$ that is part of $P_{\omega, f_{i}}^{S}$ and $V^{1}\left(P_{\omega, f_{i}}^{S}, V\right)=\varnothing$ if $\omega$ and $f_{i}$ are on the same edge. It is important to note that for two consumers $\omega$ and $\omega^{\prime}$ both on edge $e$, it is possible that $V^{1}\left(P_{\omega, f_{i}}^{S}, V\right) \neq V^{1}\left(P_{\omega^{\prime}, f_{i}}^{S}, V\right)$ for one or more values of $i$. This means that the shortest path from $\omega$ to $f_{i}$ uses one endpoint of $e$ and the shortest path from $\omega^{\prime}$ to $f_{i}$ the other. To account for this, we introduce the concept of change points.

Definition 1 A location at line segment $e_{\omega, \omega^{\prime}}$ is a change point to firm $i$, denoted $c_{\omega, \omega^{\prime}}^{i}$, if for the consumer located at $c_{\omega, \omega^{\prime}}^{i}$ there exist two different shortest paths $P_{c_{u, v}^{i}, f_{i}}^{S_{1}}$ and $P_{c_{u, v}^{i}, f_{i}}^{S_{2}}$ with

$$
d\left(P_{c_{\omega, \omega^{\prime}}^{i}, f_{i}}^{S_{1}}\right)=d\left(P_{c_{\omega, \omega^{\prime}}^{i}, f_{i}}^{S_{2}}\right)
$$

such that

$$
V^{1}\left(P_{c_{\omega, \omega^{\prime}}^{i}, f_{i}}^{S_{1}}, V\right) \neq V^{1}\left(P_{c_{\omega, \omega^{\prime}}^{i}}^{S_{2}}, f_{i}, V\right)
$$

That is, for a change point, there exist two shortest paths travel to $f_{i}$ that differ in the first vertex encountered (not including the change point when this is a vertex itself) when traveling from the change point to $f_{i}$. For example, in Figure 1, $\omega$ is a change point with respect to $f_{i}$ because there are two shortest paths differing in the first vertex encountered ( $v$ and $v^{\prime}$, respectively) when moving from $\omega$ to $f_{i}$. In contrast, although there are different shortest paths from point $d$ to $f_{i}, d$ is not a change point because for all these shortest paths, the first vertex encountered when moving from $d$ to $f_{i}$ is the same, namely $\omega$. Three lemma's regarding the existence of change points follow from this definition.

Lemma 1 Each firm has at most one change point $c_{u, v}^{i}$ per line segment $e_{\omega, \omega^{\prime}} \backslash\left\{\omega, \omega^{\prime}\right\}$.

Proof: All proofs are contained in the Appendix. 


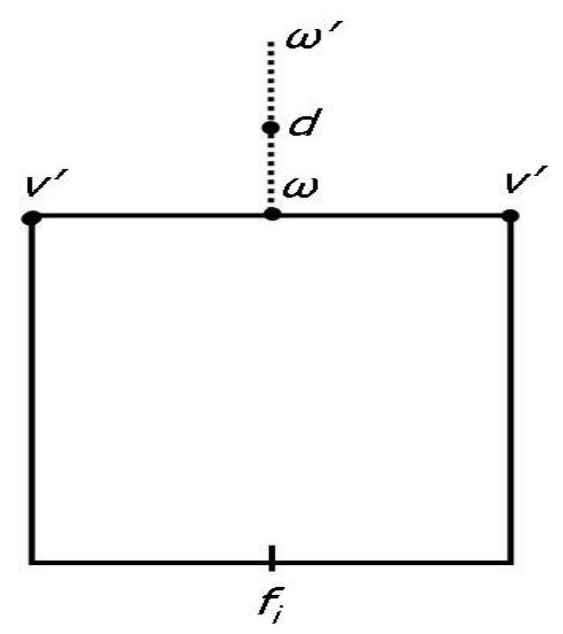

Figure 1: In graph $G=(V, E), \omega$ is a change point with respect to $f_{i}$ whereas point $d$ is not.

The lemma explicitly limits attention to the interior of line segments; line segments equal to an edge including their endpoints may inhabit multiple change points with respect to a particular firm, one in its interior and potentially one at each of the vertices $u$ and $v$. Figure 2 gives an example. Line segment $e_{\omega, \omega^{\prime}}$ has two change points with respect to firm $i$ : from the interior change point $c_{\omega, \omega^{\prime}}^{i}$, there are two shortest paths to firm $i$ (each with distance 13), one over vertex $\omega$ and the other over vertex $\omega^{\prime}$. The second change point is $c_{\omega}^{i}$ at vertex $\omega$; at this vertex that there two different shortest paths to $f_{i}$ each with distance 9 , one over vertex $\omega^{*}$ and the other over vertex $\omega^{\prime \prime}$. Lemma 1 provides an upper bound for the number of change points one has to search for: in a graph with $n$ firms and $m$ edges, the number of change points will never exceed $n m$.

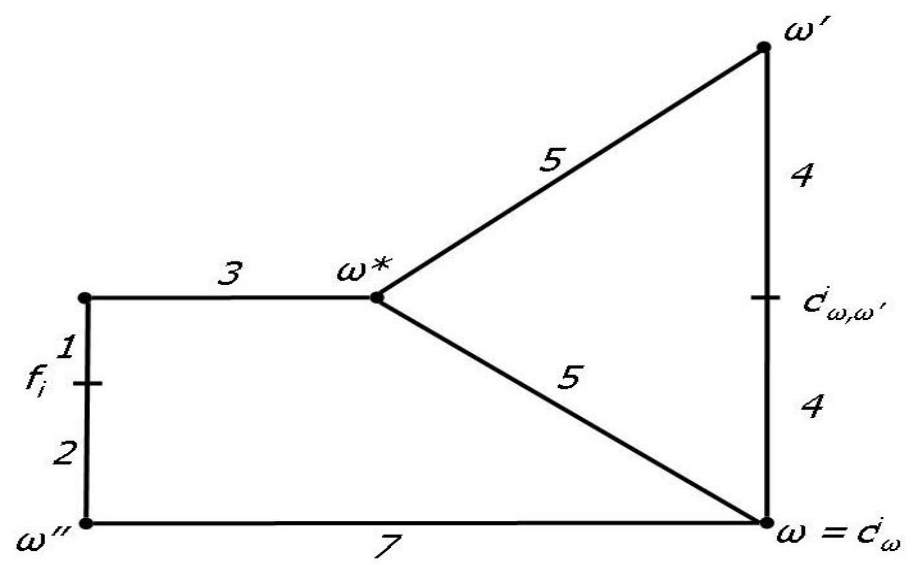

Figure 2: Example graph with multiple change points at line segment $e_{\omega, \omega^{\prime}}$. 
Lemma 2 If line segment $e_{\omega, \omega^{\prime}}$ contains a change point $c_{\omega, \omega^{\prime}}^{i}$ with respect to $f_{i}$, then $f_{i} \notin e_{\omega, \omega^{\prime}}$.

Lemma 2 implies that there is always at least one vertex between a change point of a firm and the firm itself, which ensures that the function $V^{1}(\cdot)$ in equation (1) is always defined for change points.9

\section{Lemma 3 Presence of Change Points}

A line segment $e_{\omega, \omega^{\prime}}$ has a change point with respect to firm $i$

a. in its interior if and only if

$$
f_{i} \notin e_{\omega, \omega^{\prime}} \wedge\left|d\left(P_{\omega, f_{i}}^{S}\right)-d\left(P_{\omega^{\prime}, f_{i}}^{S}\right)\right|<d\left(e_{\omega, \omega^{\prime}}\right), \text { and }
$$

$\boldsymbol{b}$. at one of its endpoints, say $\omega$, if and only if there exist vertices $v$ and $v^{\prime}$ such that

$$
\begin{gathered}
d\left(P_{\omega, f_{i}}^{S}\right)-d\left(P_{v, f_{i}}^{S}\right)=d\left(e_{\omega, v}\right) \wedge d\left(P_{\omega, f_{i}}^{S}\right)-d\left(P_{v^{\prime}, f_{i}}^{S}\right)=d\left(e_{\omega, v^{\prime}}\right) \wedge \\
\left|d\left(P_{v, f_{i}}^{S}\right)-d\left(P_{v^{\prime}, f_{i}}^{S}\right)\right|=\left|d\left(e_{\omega, v}\right)-d\left(e_{\omega, v^{\prime}}\right)\right| .
\end{gathered}
$$

The first part of Lemma 3 simply states that there is a change point with respect to firm $i$ at the interior of a line segment $e_{\omega, \omega^{\prime}}$ if and only if the difference in shortest path distance to firm $i$ at the endpoints $\omega$ and $\omega^{\prime}$ is less than the length of $e_{\omega, \omega^{\prime}}$. The second part states that an endpoint $\omega$ of a line segment is a change point if and only if two vertices (other than $\omega$ if $\omega$ is a vertex itself) can be found such that for each of these vertices, the shortest distance from the vertex to firm $i$ plus the distance from the vertex to $\omega$ equals the shortest path distance from $\omega$ to the firm and, moreover, the absolute difference in the distance from the vertices to $\omega$ must equal the absolute difference in shortest path distance from the vertices to $f_{i}$.

Figure 3 provides an illustration. The line segment $e_{v, v^{\prime}}$ contains a change point in its interior because for $\omega=v$ and $\omega^{\prime}=v^{\prime}$ the conditions under $a$ ) are satisfied. This change point is located at $u$ and any line segment on $e_{v, v^{\prime}}$ with $u$ as one of its endpoints, e.g. $e_{u, u^{\prime}}$ in Figure 3 , satisfies conditions $b$ ). It is also instructive to see that $\tilde{\omega}$ cannot be a change point for two reasons. First, according to part $a$ ) of Lemma 3 , the edge $e_{v, v^{*}}$ cannot have a change point on its interior 10 Second, for the point $\tilde{\omega}$ and vertices $v$ and $v^{\prime}$, the third condition in part $b$ ) of Lemma 3 is not satisfied (but the first two are). Lemma 3 is particularly useful because, for $\omega$ and $\omega^{\prime}$ vertices

\footnotetext{
${ }^{9}$ The fact that the edge at which a firm located will never contain a change point with respect to that firm further limits the potential number of change points to $n(m-1)$.

${ }^{10}\left|d\left(P_{v, f_{i}}^{S}\right)-d\left(P_{v^{*}, f_{i}}^{S}\right)\right|=|5-2|=3 !<d\left(e_{v, v *}\right)=3$.
} 


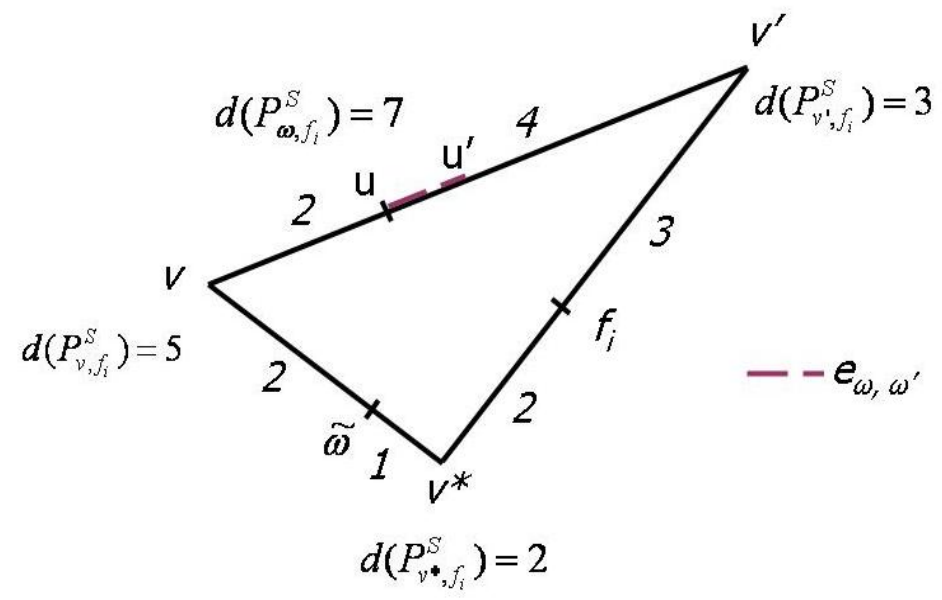

Figure 3: Illustration of Lemma 3.

of graph $G$, it gives the necessary and sufficient conditions for the presence of change points in terms of edge lengths and shortest path distances between individual vertices and firms.

For line segments $e_{\omega, \omega^{\prime}}$ with a change point $c^{i}$ with respect to firm $i$, the distance to firm $i$ is by definition increasing when moving towards $c^{i}$ and decreasing when moving away from $c^{i}$. For line segments $e_{\omega, \omega^{\prime}}$ without change point $c^{i} \in e_{\omega, \omega^{\prime}} \backslash\left\{\omega, \omega^{\prime}\right\}$, it is also useful to know whether the distance to firm $i$ is increasing or decreasing when moving from $\omega$ to $\omega^{\prime}$. To this end, we write

$$
\begin{aligned}
& d_{\omega, \omega^{\prime}}^{i}=+1 \quad \text { if } \quad d\left(\hat{\omega}, f_{i}\right)-d\left(\tilde{\omega}, f_{i}\right)>0 \forall \tilde{\omega}, \hat{\omega} \in e_{\omega, \omega^{\prime}} \text { s.t. } d(\omega, \tilde{\omega})<d(\omega, \hat{\omega}) \text {, and } \\
& d_{\omega, \omega^{\prime}}^{i}=-1 \quad \text { if } \quad d\left(\hat{\omega}, f_{i}\right)-d\left(\tilde{\omega}, f_{i}\right)<0 \forall \tilde{\omega}, \hat{\omega} \in e_{\omega, \omega^{\prime}} \text { s.t. } d(\omega, \tilde{\omega})<d(\omega, \hat{\omega}) .
\end{aligned}
$$

Thus $d_{\omega, \omega^{\prime}}^{i}=+1(-1)$ if the distance to firm $i$ is increasing (decreasing) when moving from $\omega$ to $\omega^{\prime}$.

\section{Firm-level demand}

Shortest paths and change points are all based on distances and are as such exogenous. The position of marginal consumers instead is dependent on the prices charged by the firms and as a consequence endogenous to the model. Denote the utility a consumer located at point $\omega$ derives from buying at firm $i$ by $U\left(\omega, d\left(\omega, f_{i}\right), \mathbf{p}\right)$ with $\mathbf{p}$ an $n \times 1$ price vector. I follow D'Aspremont, Gabszewicz and Thisse (1979) and assume nonlinear transportation cost. That is, we consider 
the utility function

$$
U\left(\omega, d\left(\omega, f_{i}\right), \mathbf{p}\right)=V-c d\left(\omega, f_{i}\right)^{\alpha}-p_{i}, \text { with } \alpha>1,
$$

where $c$ denotes the importance of transportation cost and $V$ is assumed large enough such that all consumers $\omega \in \Omega$ will buy in equilibrium. If a consumer buys at all, she will purchase one unit of the good. This leads to the following general quantity demanded for firm $i$ as a function of prices $\mathbf{p}$ and conditional on firm locations:

$$
q_{i}(\mathbf{p})=\left\{\omega: \omega \in G(V, E), U\left(\omega, d\left(\omega, f_{i}\right), \mathbf{p}\right) \geq U\left(\omega, d\left(\omega, f_{j}\right), \mathbf{p}\right) \forall j \neq i\right\} .
$$

The remaining part of this paper limits attention to the case with two firms $(i, j \in\{1,2\})$. To derive demand for each individual firm, we need to identify the position of the marginal consumers who are indifferent between buying at either firm for given prices. Since we consider graphs, in most cases there are multiple marginal consumers which complicates matters compared to D'Aspremont, Gabszewicz and Thisse (1979). Denote with $S_{f_{i}}$ be the set of line segments served by firm $i$, conditional on prices $p_{j}, j=1,2 . S_{f_{i}} \cap S_{f_{j}}$ is not necessarily an empty set. Let $S=\bigcup_{i} S_{f_{i}}$. The derivation on firm level demand thus boils down to determining $S_{f_{i}}$, for all firms $i$.

The algorithm we introduce determines in a systematic and computationally efficient way the position of all marginal consumers at graph $G$ and thereby firm level demand conditional on prices and location. The algorithm divides graph $G$ into different, mutually exclusive, line segments $e_{\omega, \omega^{\prime}} \backslash\left\{\omega^{\prime}\right\}$ which do not contain a change point except possibly at the point of origin $\omega$. The algorithm uses the following straightforward principle:

Proposition 4 Consider the line segment $e_{\omega, \omega^{\prime}}$, suppose that $\omega \in S_{f_{i}}$ and that there are no change points with respect to firm $i$ and $j$ on $e_{\omega, \omega^{\prime}} \backslash\left\{\omega, \omega^{\prime}\right\}$.

- If $\omega \notin S_{f_{j}}, e_{\omega, \omega^{\prime}} \backslash\{\omega\}$ contains at most one consumer y indifferent between buying from firm $i$ and buying from firm $j$ only if

$\mathbf{a}\left(d_{\omega, \omega^{\prime}}^{i}=+1\right) \wedge\left(d_{\omega, \omega^{\prime}}^{j}=-1\right) ;$

$\mathbf{b}\left(d_{\omega, \omega^{\prime}}^{i}=+1\right) \wedge\left(d_{\omega, \omega^{\prime}}^{j}=+1\right) \wedge\left(\omega \in C_{f_{j}}\right) \wedge\left(\omega \notin C_{f_{i}}\right)$;

c $\left(d_{\omega, \omega^{\prime}}^{i}=-1\right) \wedge\left(d_{\omega, \omega^{\prime}}^{j}=-1\right) \wedge\left(\omega \notin C_{f_{j}}\right) \wedge\left(\omega \in C_{f_{i}}\right)$;

- In all other cases, $e_{\omega, \omega^{\prime}} \backslash\{\omega\}$ does not inhabit an indifferent consumer. 
- If $\omega \in S_{f_{j}}$, consumers on $e_{\omega, \omega^{\prime}} \backslash\{\omega\}$ are indifferent between buying from either firm if and only if

$$
\left(d_{\omega, \omega^{\prime}}^{i}=d_{\omega, \omega^{\prime}}^{j}\right) \wedge\left(\omega \in C_{f_{i}}\right) \wedge\left(\omega \in C_{f_{j}}\right)
$$

Otherwise,

$$
\begin{gathered}
e_{\omega, \omega^{\prime}} \backslash\{\omega\} \in S_{f_{i}} \wedge e_{\omega, \omega^{\prime}} \backslash\{\omega\} \notin S_{f_{j}} \text { if } \begin{cases}d_{\omega, \omega^{\prime}}^{i}=-1 & \wedge d_{\omega, \omega^{\prime}}^{j}=+1, \text { or } \\
d_{\omega, \omega^{\prime}}^{i}=d_{\omega, \omega^{\prime}}^{j}=+1 & \wedge \omega \in C_{f_{i}} \wedge \omega \notin C_{f_{j}}, \text { or } \\
d_{\omega, \omega^{\prime}}^{i}=d_{\omega, \omega^{\prime}}^{j}=-1 \wedge \omega \notin C_{f_{i}} \wedge \omega \in C_{f_{j}} .\end{cases} \\
e_{\omega, \omega^{\prime}} \backslash\{\omega\} \notin S_{f_{i}} \wedge e_{\omega, \omega^{\prime}} \backslash\{\omega\} \in S_{f_{j}} \text { if } \begin{cases}d_{\omega, \omega^{\prime}}^{i}=+1 & \wedge d_{\omega, \omega^{\prime}}^{j}=-1, \text { or } \\
d_{\omega, \omega^{\prime}}^{i}=d_{\omega, \omega^{\prime}}^{j}=+1 & \wedge \omega \notin C_{f_{i}} \wedge \omega \in C_{f_{j}}, \text { or } \\
d_{\omega, \omega^{\prime}}^{i}=d_{\omega, \omega^{\prime}}^{j}=-1 & \wedge \omega \in C_{f_{i}} \wedge \omega \notin C_{f_{j}} .\end{cases}
\end{gathered}
$$

The condition that $\omega \in S_{f_{i}}$ and $\omega \notin S_{f_{j}}$ implies that $\omega$ itself is not the location of a marginal consumer. In situation $(a)$, the transportation cost to buy from firm $i$ is increasing when moving from $\omega$ to $\omega^{\prime}$ whereas the transportation cost to buy from firm $j$ is decreasing. In situation $(b)$, the distance to both firm $i$ and firm $j$ is increasing when moving from $\omega$ to $\omega^{\prime}$ but because the point of departure $\omega$ is closer to firm $j$ and transportation costs are assumed quadratic, transportation cost to buy from firm $i$ is increasing more rapidly than transportation cost to buy from firm $j{ }^{11}$ The opposite holds in the third situation. Here the distance to both firm $i$ as firm $j$ is decreasing when moving from $\omega$ to $\omega^{\prime}$ but since the point of departure $\omega$ is closer to firm $i$ and transportation costs are a quadratic function of distance, transportation cost to buy from firm $j$ is decreasing more rapidly than transportation cost to buy from firm $i$.

In all three cases $(a)$ to $(c)$ the implication is that there may exist a point $y \in e_{\omega, \omega^{\prime}}$ such that consumers to the left of $y$ (i.e. those closer to $\omega$ than consumer $y$ ) prefer buying from firm $i$ whereas those to the right of $y$ will buy from firm $j, e_{\omega, y} \in S_{f_{i}}$ and $e_{y, \omega^{\prime}} \in S_{f_{j}}$, with $y$ the marginal consumer indifferent between buying from either firm $i$ or firm $j$ :

$$
U\left(y, d\left(y, f_{i}\right), \mathbf{p}\right)=U\left(y, d\left(y, f_{j}\right), \mathbf{p}\right) .
$$

In case there is no indifferent consumer $y \in e_{\omega, \omega^{\prime}}$, all consumers at the line segment will buy from firm $i$, that is: $e_{\omega, \omega^{\prime}} \in S_{f_{i}}$.

The second part of the proposition states that if the point of departure $\omega$ is itself the location of an indifferent consumer, all consumers located at line segment $e_{\omega, \omega^{\prime}}$ will be indifferent too if and only if $\omega$ is at equidistance from both firms and these shortest path distances are either

\footnotetext{
${ }^{11}$ This holds more general for any $\alpha>1$.
} 
both increasing or both decreasing when moving from $\omega$ to $\omega^{\prime}: e_{\omega, \omega^{\prime}} \in S_{f_{i}}, \in S_{f_{j}}$. In all other cases, consumers on the line segment have a strict preference for buying from one of the firms. If the distance to one of the firms is decreasing when moving from $\omega$ to $\omega^{\prime}$ while it is increasing for the other firm, consumers prefer to buy from the firm the distance to which is decreasing. If the distance to both firms is decreasing (increasing) when moving from $\omega$ to $\omega^{\prime}$, consumers prefer to buy from the firm whose distance to the source vertex is largest (smallest).

We have the following corollary for line segments $e_{\omega, \omega^{\prime}}$ with $\omega \in S_{f_{i}}$ that contain a change point with respect to firm $j \neq i$.

Corollary 5 Let line segment $e_{\omega, \omega^{\prime}}$ contain a change point $c$ with respect to $f_{j}, \omega \in S_{f_{i}}^{e}$ and $\omega \in C_{f_{i}}$.

$$
\text { If } \omega \notin S_{f_{j}}, e_{\omega, c} \in S_{f_{i}} \text {. }
$$

If $\omega \in S_{f_{j}}$, consumers on $e_{\omega, c} \backslash\{\omega\}$ are indifferent between buying from either firm if and only if $\left(d_{\omega, \omega^{\prime}}^{i}=+1\right) \wedge\left(\omega \in C_{f_{i}}\right) \wedge\left(\omega \in C_{f_{j}}\right)$.

The corollary tells us that for a line segment $e_{\omega, \omega^{\prime}}$ with $\omega$ served exclusively by firm $i$, all consumers located between $\omega$ and change point $c^{j}$ with respect to firm $j$ will prefer buying from firm $i$ to buying from firm $j$. The second part gives conditions for these consumers to be indifferent, given that consumers at point $\omega$ are indifferent. We now continue with the algorithm which determines demand given firms' locations and prices.

\subsection{The demand algorithm for two firms}

In what follows, consumers at edge or line segment $e_{\omega, \omega^{\prime}}$ indifferent between buying from either firm $i$ or $j$ are denoted as $y_{\omega, \omega^{\prime}}^{i, j}$ and the position of a change point at $e_{\omega, \omega^{\prime}}$ with respect to firm $j$ is denoted as $c_{\omega, \omega^{\prime}}^{j}$. With $y^{f_{i}, f_{j}}$ we denote the indifferent consumer located (if anywhere) along the shortest path between firm $i$ and $j$, and with $y^{f_{i}}\left(y^{f_{j}}\right)$ the indifferent consumer located (if anywhere) on a part of the home edge of firm $i(j)$ not included in the shortest path.

Given a graph $G=(V, E)$ and given the position of firms 1 and 2 in $G$, first calculate

- The shortest path $P_{v, v^{\prime}}^{S}$ for all unordered pairs of vertices $\left(v, v^{\prime}\right) \in V$

\footnotetext{
${ }^{12}$ The Floyd-Warshall algorithm (see e.g. Bertsekas, 1991, p. 82-83) calculates the shortest path from each vertex to each other vertex. $P_{v, v^{\prime}}^{S}$ minimizes $\sum_{p \in P} d(p)$ among all paths connecting $v$ and $v^{\prime}$.
} 
- the shortest path $P_{f_{1}, f_{2}}^{S}$ connecting $f_{1}$ and $f_{2}$;

- for all vertices $v \in V$ and firms $i=1,2$ whether $v \in C_{f_{i}}$;

- for each edge $e_{u, v} \in E$ whether it inhabits any change points $c_{v}^{i}$ and $c_{u, v}^{i}, i=1,2$.

In the first step, the location of the marginal consumer (if any) along the shortest path between firms $f_{1}$ and $f_{2}$ is determined. This gives us an initial set $S$ of locations for which we know by which firm each point is served ${ }^{13}$ In each subsequent step, this set is expanded by adding to $S$ all edges which have at least one endpoint, say $\omega$, in $S$. This is repeated until $S=G$. Note that the algorithm does not terminate before $S=G$ whenever $G$ is a connected graph. Because $\omega$ is in $S$, we know for this source vertex to which firm(s) it is closest and by which firm(s) the point is served and we can apply Proposition 4 and Corollary 5 from which firm each consumer at $e_{\omega, \omega^{\prime}}$ will buy. The demand algorithm reads as follows:

\section{FIRM LEVEL DEMAND ALGORITHM}

0. Set $S_{f_{i}}^{0}=\varnothing \forall i . S^{0}=\bigcup_{i} S_{f_{i}}^{0}$.

1. Let $t=1$. Consider all edges $e_{u, v}$ such that $e_{u, v} \in P_{f_{1}, f_{2}}^{S} \vee e_{u, v}=e^{f_{1}} \vee e_{u, v}=e^{f_{2}}$.

a. Determine whether $y^{f_{1}, f_{2}} \in P_{f_{1}, f_{2}}^{S}, y^{f_{1}, f_{2}} \in e^{f_{1}}$ or $y^{f_{1}, f_{2}} \in e^{f_{2}}$, if any. This determines for each consumer $\omega \in P_{f_{1}, f_{2}}^{S}$ whether $\omega \in S_{f_{i}}^{1}$ or $\omega \notin S_{f_{i}}^{1}$ for all firms $i$.

b. For $i=1,2$ :

i if $e^{f_{i}}$ does not inhabit a change point with respect to firm $3-i, y^{f_{1}, f_{2}}$ determines for each consumer $\omega \in e^{f_{i}}$ whether $\omega \in S_{f_{j}}^{1}$ or $\omega \notin S_{f_{j}}^{1}$ for all firms $j$.

ii if $e^{f_{i}}$ inhabits a change point with respect to firm $3-i$, subdivide the edge in the line segments $e_{f_{i}, c_{f_{i}}^{1}}$, and $e_{c_{f_{i}}^{1}, v}$, with $v$ the vertex of $e^{f_{i}}$ that does not belong to $P_{f_{1}, f_{2}}^{S}$. Determine for each of these line segments $e_{u, v}$ the location of an indifferent consumer $y_{u, v}^{i, j}$ (if any) using Proposition 4. This determines for each consumer $\omega \in e_{u, v}$ whether $\omega \in S_{f_{i}}^{1}$ or $\omega \notin S_{f_{i}}^{1}$ for all firms $i$.

\footnotetext{
${ }^{13}$ Note that there can be change points at $P_{f_{1}, f_{2}}^{S}$, but these can only occur at the vertices because by the definition of a shortest path, the distance to firm $i$ must always increase when one moves over $P_{f_{1}, f_{2}}^{S}$ towards $f_{2}$. For this reason, these change points are irrelevant in determining firm level demand from consumers at $P_{f_{1}, f_{2}}^{S}$. An example is a graph in the form of a kite with firm 2 located at the rope and firm 1 at the top of the kite. This graph has a change point with respect to firm 1 at the vertex where the rope is attached to the kite. Firms can also have a change point on the part of the home edge of the other firm that does not belong to the shortest path.
} 
c. $S^{1}=S^{0} \cup\left(\bigcup_{i} S_{f_{i}}^{1}\right)$.

2. Set $t=t+1$. Consider all edges $e_{u, v}$ with $e_{u, v} \backslash\{u, v\} \notin S^{t-1}$ and $u \in S^{t-1}$.

a. If $e_{u, v}$ does not contain a change point, determine the location of $y_{u, v}^{i, j}$ (if any) using Proposition 4. This determines for each consumer $\omega \in e_{u, v}$ whether $\omega \in S_{f_{i}}^{t}$ or $\omega \notin S_{f_{i}}^{t}$ for all firms $i$.

b. If $e_{u, v}$ inhabits $k \geq 1$ change points, subdivide the edge in the line segments $e_{u, c_{u, v}}^{i_{1}}$, $e_{c_{u, v}^{i_{1}, c_{u, v}} i_{2}}, \ldots, e_{c_{u, v}^{i_{k-1}, c_{u, v}}}^{i_{k}}, e_{c_{u, v}^{i_{k}, v}}$ and perform step $2 a$ for each line segment separately by considering first $e_{u, c_{u, v}^{i_{1}}}$, then $e_{c_{u, v}^{i_{1}}, c_{u, v}^{i_{2}}}$ and so on until $e_{c_{u, v}^{i_{k}, v}}$.

c. $S^{t}=S^{t-1} \cup\left(\bigcup_{i} S_{f_{i}}^{t}\right)$.

3. Repeat step 2 until $S=G$.

\subsection{Example}

Consider the simple graph $G=(V, E)$ depicted in Figure 4 with the locations of firm 1 and 2 denoted by $f_{1}$ and $f_{2}$. Consumers are uniformly distributed along the edges and vertices of the graph and face quadratic transportation cost. In this graph, $e_{1,2}$ is the home edge of firm 1 and $e_{3,4}$ is the home edge of firm 2. $P_{f_{1}, f_{2}}^{S}=\left(f_{1}-v_{2}\right) e_{2,3} v_{3}\left(v_{3}-f_{2}\right) ; V\left(P_{f_{1}, f_{2}}^{S}\right)=\left\{v_{2}, v_{3}\right\}$; $C_{f_{1}}=\{1,2,6,8,9\}$ and $C_{f_{2}}=\{3,4,5,7,10\}$. For the moment, suppose that firm 1 charges a price $p_{1}=20$ and firm 2 a price $p_{2}=10$.

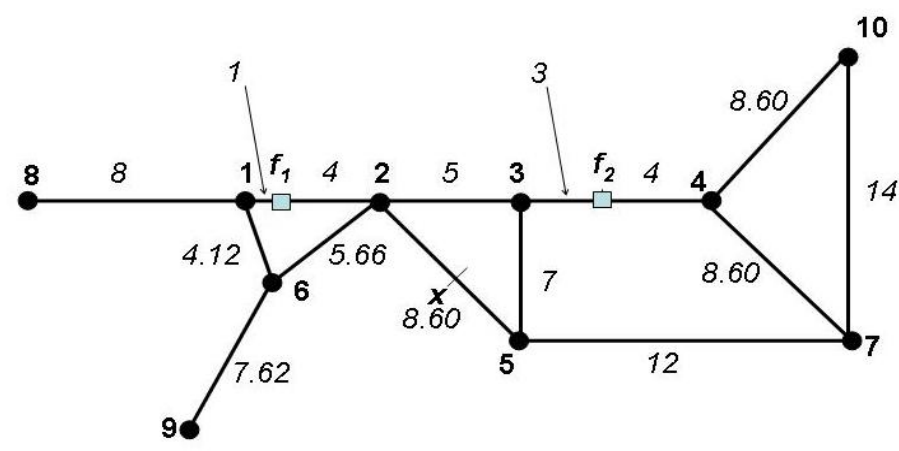

Figure 4: Example graph $G=(V, E)$ with uniformly distributed consumers and two firms $f_{1}$ and $f_{2}$. Edge lengths in italics.

For the consumer located at point $x, P_{x, f_{1}}^{S}=x-v_{2} v_{2}-f_{1}$ and $P_{x, f_{2}}^{S}=x-v_{5} v_{3} v_{3}-f_{2}$, thus $V^{1}\left(P_{x, f_{1}}^{S}\right)=\{2\}$ and $V^{1}\left(P_{x, f_{2}}^{S}\right)=\{5\}$ 
The change points for firm 1 and firm 2 are depicted in Figure 5. Firm 1 has four change points: $c_{2,6}^{1}$ at edge $e_{2,6} ; c_{3,5}^{1}$ at edge $e_{3,5}, c_{7,10}^{1}$ at edge $e_{7,10}$ and $c_{7}^{1}$ at vertex 7 . For example, at point $c_{7}^{1}$, the distance to firm 1 via vertex 4 and 5 are equal. Firm 2 also has four change points: $c_{1,6}^{2}, c_{2,5}^{2}, c_{5,7}^{2}$ and $c_{7,10}^{2}$.

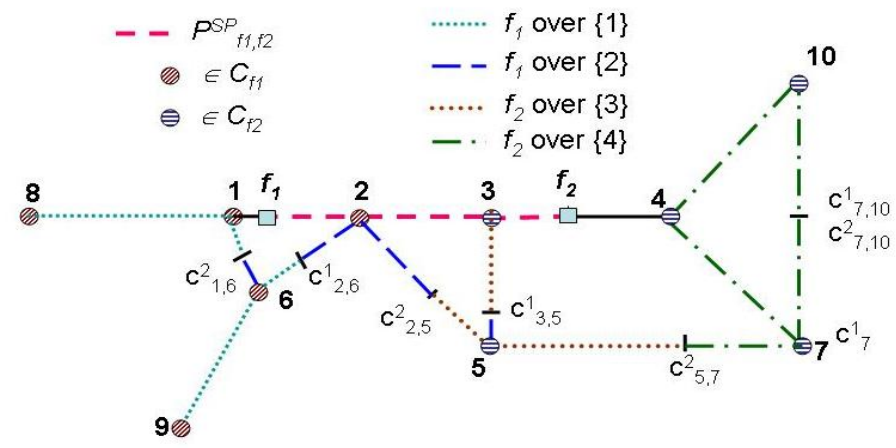

Figure 5: Change points and shortest path between $f_{1}$ and $f_{2}$ in graph $G=(V, E)$.

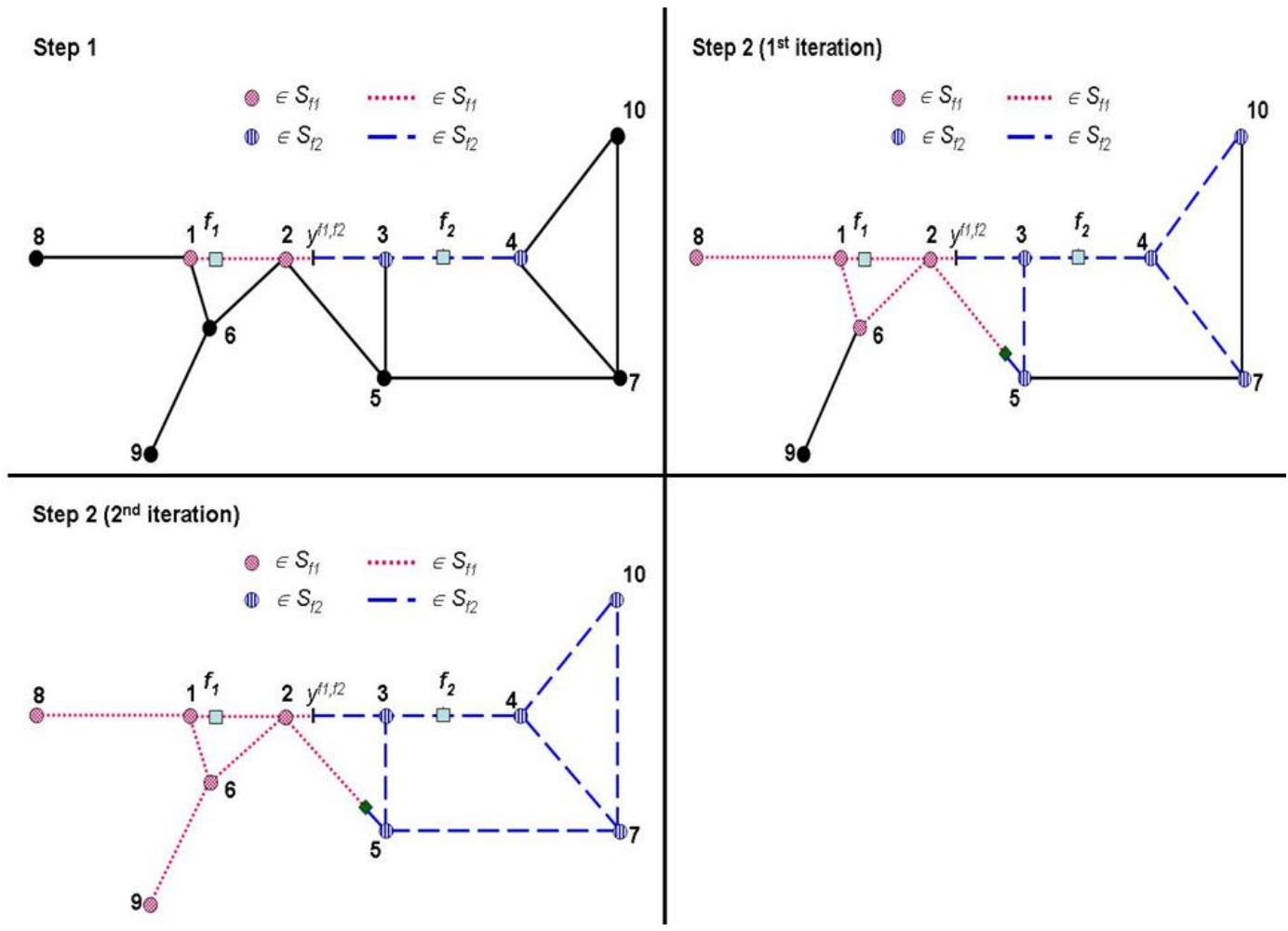

Figure 6: Illustration of the subsequent steps of the demand algorithm, conditional on $p_{1}=p_{2}$. Consumers located at the dashed lines buy from firm 2, consumers at the dotted lines buy from firm 1. 
Step 1 First we determine from which firm the consumers along $P_{f_{1}, f_{2}}^{S}$ buy. This situation is akin to the problem analyzed by D'Aspremont et al. (1979): if the marginal consumer $y_{f_{2}}^{f_{1}}$ is located at $P_{f_{1}, f_{2}}^{S}$, consumers on the path $P\left[f_{1}, y_{f_{2}}^{f_{1}}\right)$ prefer buying from firm 1 and those on the path $P\left(y_{f_{2}}^{f_{1}}, f_{2}\right]$ prefer buying from firm 2; if $y_{f_{2}}^{f_{1}} \notin P_{f_{1}, f_{2}}^{S}$ and $d_{y_{f_{2}}, f_{1}}<(>) d_{y_{f_{2}}, f_{2}}$, all consumers will buy from firm 2 (firm 1). Figure 6 shows the position of the marginal consumer $y_{f_{2}}^{f_{1}}$ in the example graph along the shortest path between the two firms, given that $p_{1}=p_{2}$. When firms charge the same price, the marginal consumer is naturally located midway between firm 1 and 2. We have:

- $e_{1,2}, e_{2,3}, e_{3, y^{1,2}} \in S_{f_{1}}^{1}$ and $e_{y_{3,4}^{1,2}, 4} \in S_{f_{2}}^{1}$ and $S^{1}=S^{0} \cup\left(\bigcup_{i} S_{f_{i}}^{1}\right)$.

Step 2 - Iteration 1 There are seven edges with one of their endpoints in $S^{1}: e_{1,8}, e_{1,6}, e_{2,6}$, $e_{2,5}, e_{3,5}, e_{4,7}$ and $e_{4,10}$. First consider the edge $e_{1,8}$ with vertex 1 as one of its endpoints. This edge does not have change points and vertex $v_{1} \in S_{f_{1}}, v_{1} \notin S_{f_{2}}$. Because $\left(d_{v_{1}, v_{8}}^{1}=+1\right) \wedge\left(d_{v_{1}, v_{8}}^{2}=\right.$ $+1)$ and $v_{1} \in C_{f_{1}} \wedge v_{1} \notin C_{f_{2}}$, Proposition 4 states that edge $e_{1,8}$ does not contain an indifferent consumer. For this reason, $e_{1,8} \in S_{f_{1}}^{2}$. Next consider edge $e_{1,6}$ which contains a change point $c_{1,6}^{2}$ with respect to firm 2 . The edge is therefore split in the line segments $v_{1}-c_{1,6}^{2}$ and $c_{1,6}^{2}-v_{6}$. For the first line segment, $\left(d_{v_{1}, c_{1,6}^{1}}^{1}=+1\right) \wedge\left(d_{v_{1}, c_{1,6}^{2}}^{2}=+1\right)$ and demand on this segment thus falls to firm 1 according to Proposition 4 . For the second segment, $\left(d_{c_{1,6}^{1}, v_{2}}^{1}=+1\right) \wedge\left(d_{c_{1,6}^{2}, v_{2}}^{2}=-1\right)$ and this segment thus may contain a consumer indifferent between buying from either firm 1 or 2. It however turns out that there is not, such that $c_{1,6}^{2}-v_{6} \in S_{f_{1}}^{2}$. Next consider edge $e_{2,6}$. Vertex $2 \in S_{f_{1}}$ and $\notin S_{f_{2}}$. Edge $e_{2,6}$ contains a change point $c_{2,6}^{1}$ with respect to firm 1 and we subdivide the edge into the line segments $v_{2}-c_{2,6}^{1}$ and $c_{2,6}^{1}-v_{6}$. Both $v_{2}$ and $c_{2,6}^{1} \in C_{f_{1}}$ and $\notin C_{f_{2}}$. Since $\left(d_{v_{2}, c_{2,6}^{1}}^{1}=+1\right) \wedge\left(d_{v_{2}, c_{2,6}^{2}}^{2}=+1\right)$ and $\left(d_{c_{2,6}^{1}}^{1}, v_{6}=-1\right) \wedge\left(d_{c_{2,6}^{2}, v_{6}}^{2}=+1\right)$, Proposition 4 says that neither segment contains an indifferent consumer, such that $e_{2,6} \in S_{f_{1}}^{1}$. Results for the remaining four edges are summarized below:

$e_{2,5} \quad v_{2} \in S_{f_{1}}, \in C_{f_{1}}$ and $\notin S_{f_{2}}, \notin C_{f_{2}}$. One change point $c_{2,5}^{2}$; subdivision into line segments $v_{2}-c_{2,5}^{2}$ and $c_{2,5}^{2}-v_{5} \cdot\left(d_{v_{2}, c_{2,5}^{2}}^{5}=+1\right) \wedge\left(d_{v_{2}, c_{2,5}^{2}}^{2}=+1\right)$, no indifferent consumer at $v_{2}-c_{2,5}^{2}$. $\left(d_{c_{2,5}^{2}, v_{6}}^{1}=+1\right) \wedge\left(d_{c_{2,5}^{2}, v_{6}}^{2}=-1\right)$. There is a potential indifferent consumer at $c_{2,5}^{2}-v_{6}$ according to Proposition 4(a). Calculation shows that this consumer $y_{c_{2,5}^{2}}^{v_{6}}$ indeed exists. Thus $v_{2}-y_{c_{2,5}^{2}}^{v_{6}} \in S_{f_{1}}^{2}$ and $y_{c_{2,5}^{2}}^{v_{6}}-v_{6} \in S_{f_{1}}^{2}$

$e_{3,5} \quad v_{3} \in S_{f_{2}}, \in C_{f_{2}}$ and $\notin S_{f_{1}}, \notin C_{f_{1}}$. One change point $c_{3,5}^{1}$; subdivision into line segments 
$v_{3}-c_{3,5}^{1}$ and $c_{3,5}^{1}-v_{5} \cdot\left(d_{v_{3}, c_{3,5}^{1}}^{5}=+1\right) \wedge\left(d_{v_{3}, c_{3,5}^{1}}^{2}=+1\right)$, no indifferent consumer at $v_{3}-c_{3,5}^{1}$. $\left(d_{c_{3,5}^{1}, v_{5}}^{1}=-1\right) \wedge\left(d_{c_{3,5}^{1}, v_{5}}^{2}=+1\right)$. There is a potential indifferent consumer at $c_{2,5}^{2}-v_{6}$ according to Proposition 4(a). Calculation shows that this consumer does not exist. Thus $e_{3,5} \in S_{f_{2}}^{2}$.

$e_{4,7}$ By similar reasoning as for $e_{1,8}$, no indifferent consumers along this edge and $e_{4,7} \in S_{f_{2}}^{2}$.

$e_{4,10}$ Idem.

See the upper right panel of Figure 6 for an illustration.

Step 2 - Iteration 2 The remaining edges $e_{6,9}, e_{5,7}$ and $e_{7,10}$ are added in the next iteration $(t=3)$ of step 2. It turns out that for the posted prices, none of them contains an indifferent consumer. The bottom left panel of Figure 6 shows firm level demand for both firms in this case.

\subsection{Spatial discontinuities in firm level demand}

In situations where firms charge the same price and the entire market is covered, each consumer buys at the firm to which she is closest and an individual firm's customers are simply the agents who are closer to this firm than to any other firm. As Figure 6 shows, a firm's customers base is a connected group of agents. It is instructive to notice that when firms charge different prices, this no longer has to hold and the spatial distribution of firm level demand may be less intuitive. Figure 7 shows the case where $p_{1}=50$ and $p_{2}=10$. Compared to Figure 6 , firm 1 now charges a higher price and receives less demand as a result. Remarkably however, some consumers at edge $e_{2,5}$ prefer buying from firm 1 , while being surrounded by consumers who have a preference for firm 2's product. The example thus gives the following result:

Result 6 The two-firm graph model of price competition may lead to spatial discontinuities in firm level demand when transportation cost are quadratic.

The intuition behind this result is that although firm 2 is cheaper, these consumers are closer to firm 1. Since distance enters quadratically in the utility function for these consumers the difference in distance outweighs the price difference. For the other agents on $e_{2,5}$, the price difference outweighs the difference in distance. The possibility of observing spatial discontinuities in an individual firm's demand distinguishes price competition on graphs from the common line and circular models like D'Aspremont et al. (1979) and Salop (1979). 


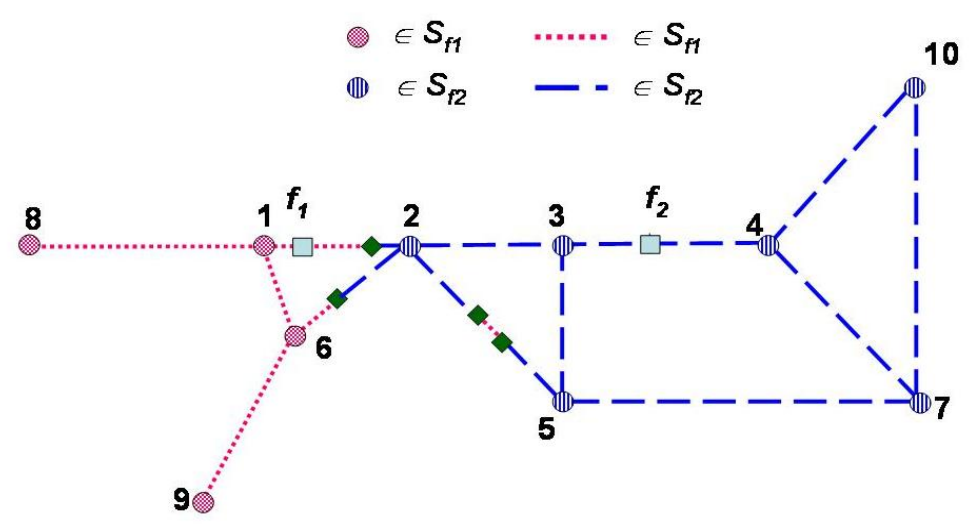

Figure 7: Illustration of spatial discontinuities in firm-level demand, $p_{1}=50 ; p_{2}=10$. Consumers located at the dashed lines buy from firm 2, consumers at the dotted lines buy from firm 1.

\section{Equilibrium existence}

\subsection{Pure strategy price equilibria}

This section will illuminate the conditions that need to hold for a graph with given firm locations to have a unique pure Nash-equilibrium in prices. I show by example that even with quadratic transportation cost, there is no clear-cut relation between equilibrium existence and graph characteristics such as the number of nodes or the degree of individual nodes. This motivates me to introduce the concepts of demand plateaus and (un)shielded hinterlands that allow me to extend the idea of "hinterlands" to graphs. It turns out that equilibrium existence is closely related to the presence of unshielded hinterlands.

It is insightful to return to D'Aspremont et al. (1979) and to discuss the intuition behind their result that the version of the price competition model with quadratic transportation costs has a unique equilibrium in pure strategies whereas the model with linear transportation costs as proposed by Hoteling (1929) has not. To this end, I introduce a distinction between shielded and unshielded hinterlands.

\section{Definition 2 Shielded Hinterland}

The shielded hinterland of firm $i$ is the set of consumers whose shortest path to any firm $j \neq i$ leads through the location of firm $i$.

This definition of shielded hinterland is what in most textbooks (e.g. Martin, 2002) is defined (implicitly) as the hinterland for firm $i$. In the classic Hotelling line, depicted in Figure 8 (neglect 
node $D$ for the moment), firm 1's hinterland are the set of consumers located between endpoint $A$ and firm 1, and firm 2's hinterland are the consumers between firm 2 and endpoint $B$. Firm $i$ can increase its hinterland by moving closer to its competitor but in doing so, the less this opponent has to decrease price to capture (part of) the hinterland of firm $i$. However, with quadratic transportation cost, the hinterland captured for a given price decrease is less than with linear transportation cost.

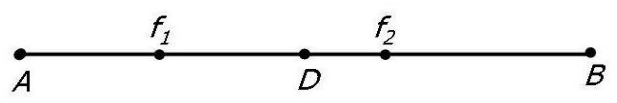

Figure 8: The complete bipartite $K_{1,2}$ graph: The Hotelling line.

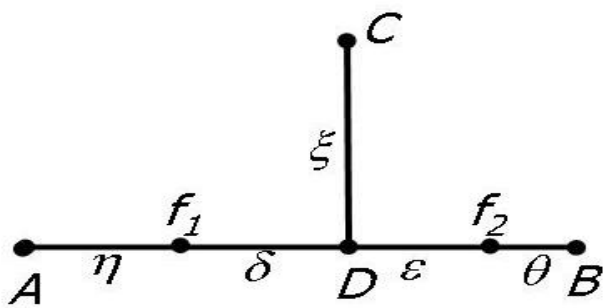

Figure 9: The complete bipartite $K_{1,3}$ graph: The Hotelling line with a junction.

In graph-theoretic terms, the Hotelling line as drawn in Figure 8 is a complete bipartite $K_{1,2}$ graph, that is, a tree with one internal node and 2 leaves (edges) 14 The most straightforward extension of the Hotelling line is the $K_{1,3}$ graph depicted in Figure 9, a star graph that more informally can be described as a "Hotelling line with a junction". To deal with graphs, I generalize the definition of hinterland such that it also covers patches of consumers not necessarily behind one firm as seen from the perspective of its competitor. It is useful to introduce the concept of demand plateaus before giving the formal definition of these unshielded hinterlands 15

\footnotetext{
${ }^{14} \mathrm{~A}$ graph $G$ is bipartite if its vertices can be divided into two classes $H_{1}$ and $H_{2}$ such that $H_{1} \cap H_{2}=\emptyset$ and $H_{1} \cup H_{2}=V(G)$ and every edge joins a vertex of $H_{1}$ to a vertex of $H_{2}$. A bipartite graph $G$ is called a complete bipartite graph is the graph contains all possible edges joining edges in the two distinct classes. Star graphs with $k+1$ nodes are complete bipartite graphs with $k$ leaves and are also denoted as $S_{k}$. The star $S_{3}$ (or $K_{1,3}$ ) with three edges is also called a claw. Because node $D$ in Figure 8 serves no real purpose and can be left out, the Hotelling line may as well be described as a $K_{1,1}$ graph: two nodes joined by one edge. See e.g. Bollobás (1998) for a formal treatment of graph theory.

${ }^{15}$ The name 'plateau' is inspired by the three-dimensional graphs with the difference in distance to firm 1 and 2 on the $z$-axis.
} 


\section{Definition 3 Demand plateau}

A demand plateau $\check{D}_{i j}$ between firm $i$ and $j$ is a compact set of consumers each of whom has the same difference in distance to firm $i$ and firm $j$.

For a market with two firms, the difference in travel distance to firm 1 and 2 is the same all consumers on the plateau, which means that once a firm has lowered price enough to make the customer at one end of the plateau buy from him, only a relatively small further decrease is needed to gain more market share at the plateau. In the extreme case of linear transportation cost, when a firm undercuts enough to win one consumer of the plateau, the firm by definition receives demand from all consumers of the plateau.

\section{Definition 4 Unshielded hinterland}

The unshielded hinterland of firm $i$ consists of the union of the demand plateaus $\check{D}_{i k}$ for which the following two conditions hold:

$i$ the consumers in demand plateau $\check{D}_{i k}$ are at least as close to firm $i$ than to any of the other firms;

ii for all consumers in plateau $\check{D}_{i k}$, the shortest path to firm $k$ does not lead through the location of firm $i$.

The shielded hinterlands in Figure 8 are both examples of demand plateaus. In the case of a line with two firms, the two definitions coincide as all hinterlands are also shielded hinterlands. This does not hold for graphs. To see this consider the simple four-node graph in Figure 9 (ignore the Greek letters). The consumers on the line $C-D$ are not a shielded but an unshielded hinterland to firm 2: these consumers form a compact set, the difference in distance to the two firms is the same for all consumers in the set and all consumers are closer to firm 2 than to firm 1 , whereas their shortest path to firm 1 does not include the location of firm 2 . The concept of hinterland is generalized as follows:

\section{Definition 5 Hinterland}

The hinterland of firm $i$ consists of the union of demand plateaus $\check{D}_{i k}$ of which the consumers are as least as close to firm $i$ than to any of the other firms.

In other words, the hinterland of firm $i$ consists of the union of its shielded and unshielded hinterlands. In Figure 9, the hinterland of firm 2 are the consumers at line segment $e_{f_{2}, B}$ and 

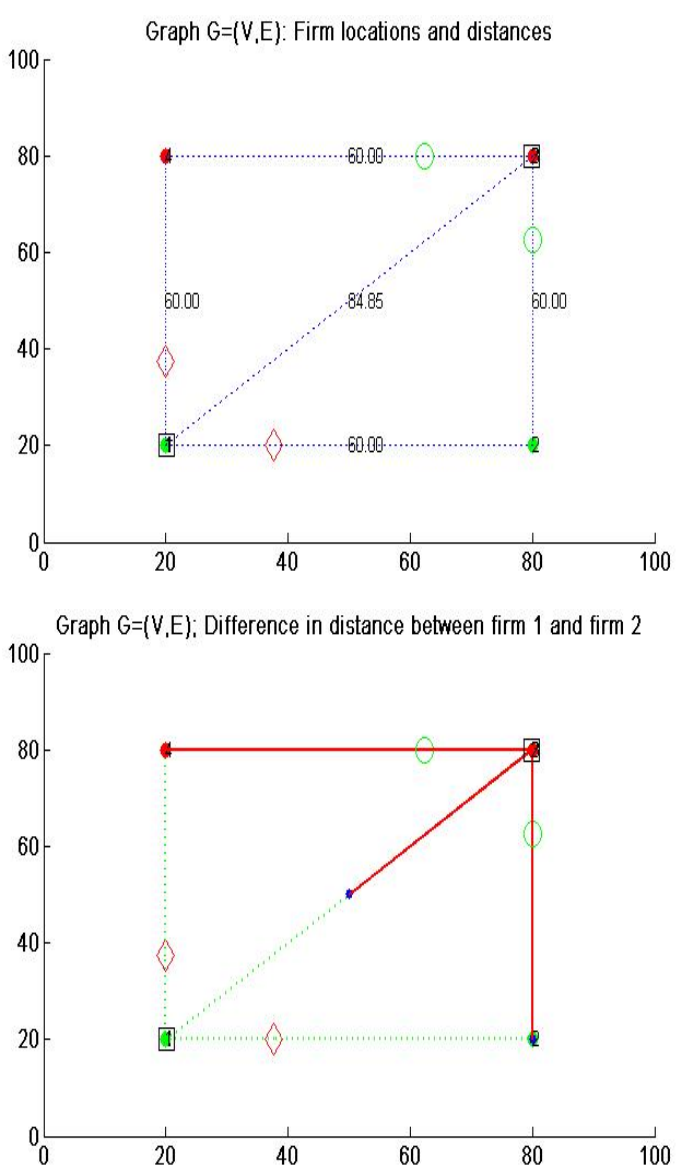

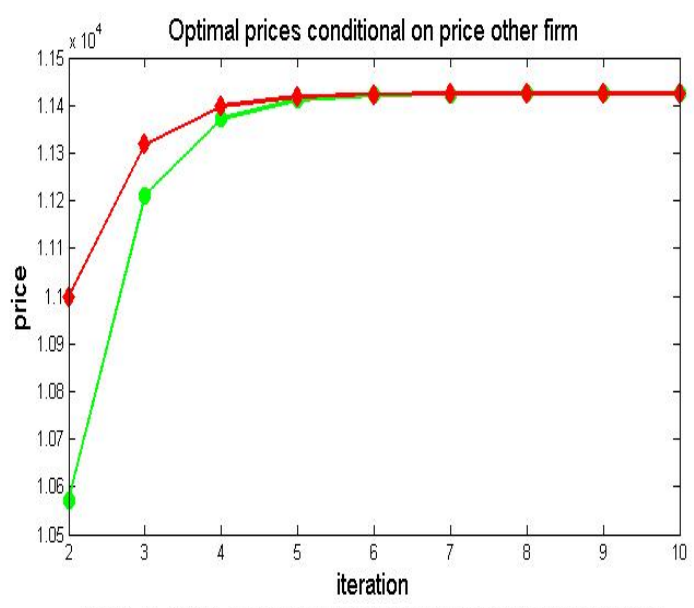

Graph $G=(V, E)$; Difference in distance between firm 1 and firm 2

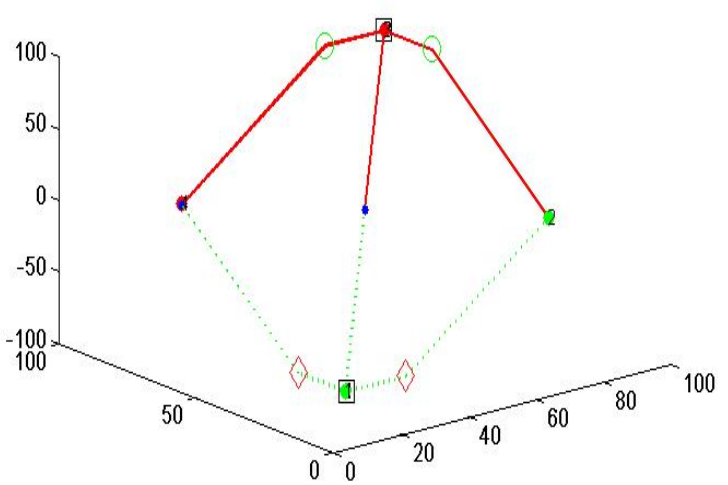

Figure 10: Four node structure with firms located at nodes with degree 3 (upper-left panel). The upper right panel depicts the resulting price dynamics. The bottom panels shows equilibrium firm level demand in two (bottom-left) and three dimensions (bottom right)

those located along edge $e_{C D}$, but not the consumers at line segment $e_{D, f_{2}}$. To reiterate, the definition of hinterland conveys the idea that for firm 2, once it has lowered price far enough to receive demand from the consumer at point $D$, it is relatively easy, and therefore attractive, to try to capture all demand originating from the line $C-D$. The reason is that when one starts at point $f_{2}$, once one has reached point $D$ and continues to travel along $e_{C D}$, the distance to firm 1 is no longer decreasing 16

It is easy to show by example that - given our formulation where consumers reside on the edges - any attempt to relate equilibrium existence and price dynamics to general characteristics of the graph (e.g. its connectivity and degree distribution) are doomed to be unfruitful. For example, consider the four node structure in the upper-left panel of Figure 10. This graph is

\footnotetext{
${ }^{16}$ This property of hinterlands holds as well for models with non-quadratic transportation costs. In case of linear transportation costs for example, when the consumers at point $D$ buy from firm 2, all consumers along the line $e_{C D}$ will buy from firm 2 .
} 

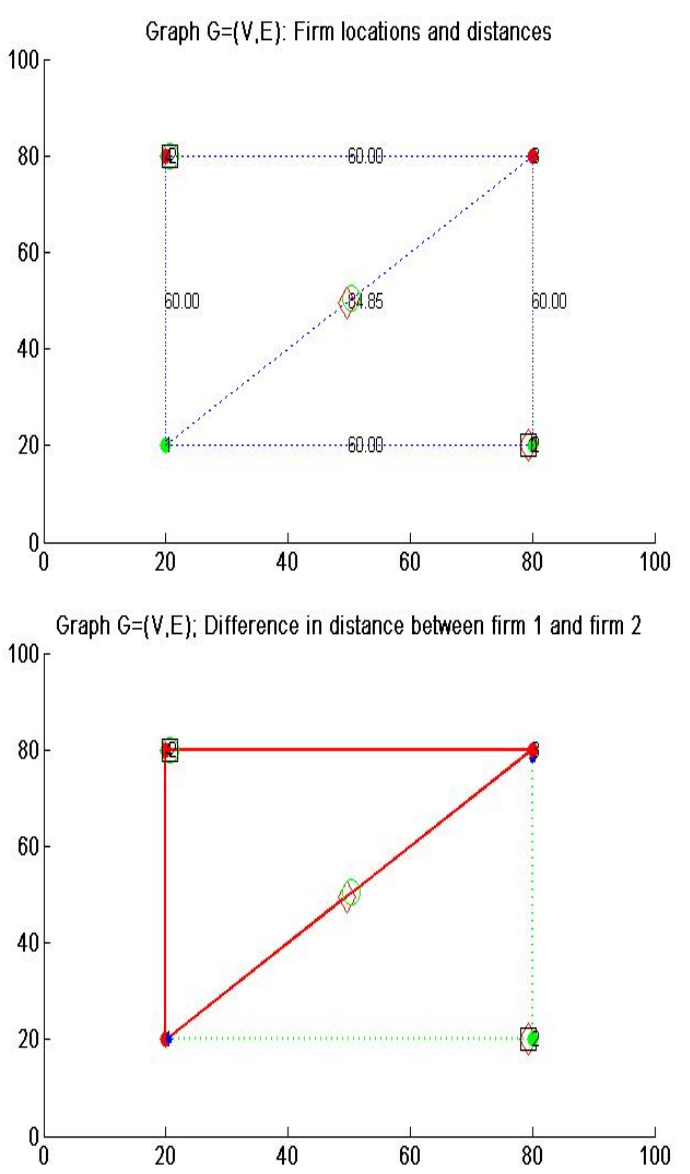

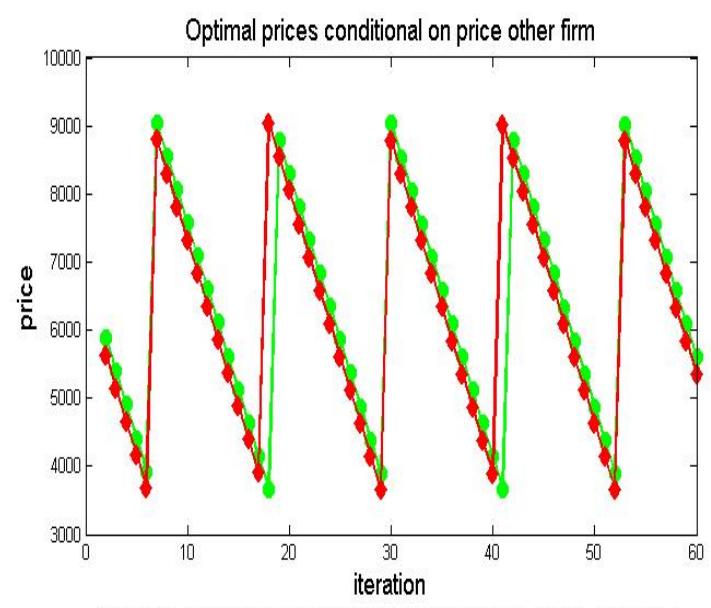

Graph $G=(V, E)$; Difference in distance between firm 1 and firm 2

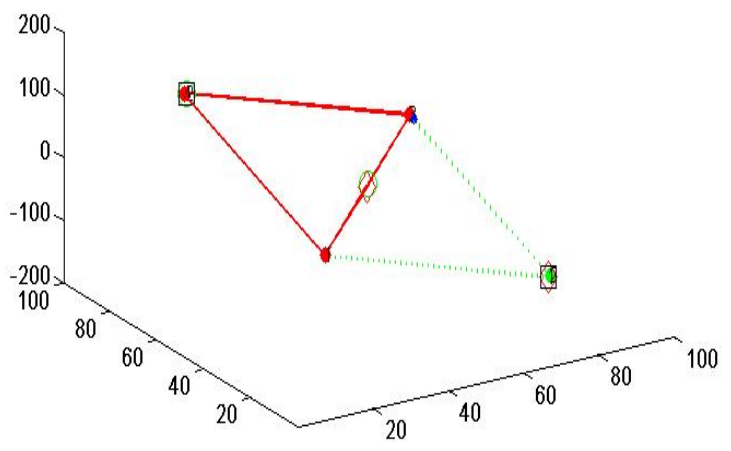

Figure 11: Four node structure with firms located at nodes with degree 3 (upper-left panel). The upper right panel depicts the resulting price dynamics. The bottom panels shows equilibrium firm level demand in two (bottom-left) and three dimensions (bottom right)

complete, apart from the edge between nodes 2 and 4 . Firms 1 and 2 are located at nodes 1 and 3, respectively. The other panels shows that, when firms follow a Markov alternating move price adjustment process, prices converge to an unique equilibrium in a small number of steps $\left(p_{1}^{*}=p_{2}^{*} \approx 11417\right)$. The bottom panels show that in equilibrium, demand is split between the two firms and each firm earns about $11417 / 2=5508$. In fact, the bottom-right panel of Figure 10 illustrates that this graph is very similar to the familiar Hotelling model with consumers on a unit interval. Other than in the model discussed by D'Aspremont et al., consumers are non-uniformly distributed but their density is somewhat higher towards the middle of the line.

Now consider the same graph but with the two firms located at nodes 2 and 4 (Figure 11). In this case, there is no price equilibrium in pure strategies. The upper-right panel of this Figure 11 shows that long periods of undercutting by relatively small amounts are followed by a price hike. The reason is that, in this configuration of graph and firm locations, there is a 
considerable unshielded hinterland: the consumers located at the edge connecting nodes 1 and $3{ }^{17}$ For both firms, it is attractive to undercut the other firm to receive demand from these consumers, until prices have decreased to the point that for one of the firms it is more attractive to retreat and to raise prices. That the existence of a price equilibrium in pure strategies can be elusive is also illustrated by the two pentagons in Figure 12. The only difference between the two graphs is that the position of firm 1 in the right pentagon is somewhat closer to node 5. Whereas the first configuration has a pure-strategy price equilibrium, the second, almost identical configuration has not.

D'Aspremont et al. (1979) have shown that in the linear Hotelling model, price equilibria are not defined for some positions of the firms on the line (if they are too close), but that these are defined for all possible firm locations when transportation costs are quadratic. The examples above show that for graphs, imposing quadratic costs is not sufficient to guarantee price equilibria in pure strategies.

We thus have that:

Result 7 In graph models of price competition, the assumption of quadratic transportation cost is not sufficient for the existence of pure-strategy price equilibria.

Contrast this result with Economides (1986a) who shows that with consumers uniformly distributed on the unit interval with utility described by (2), firms will in equilibrium maximally differentiate whenever $\alpha \geq 5 / 3^{18}$ In the graph depicted in Figure 11, firms are maximally differentiated (they cannot be further apart), $\alpha=2>5 / 3$, but no equilibrium exists. In particular for $K_{1,3}$ graph, the most straightforward extension of the Hotelling line (depicted in Figure 9), one can prove the following:

Theorem 8 For every $K_{1,3}$ graph, there exists a configuration of firm locations for which the price competition game does not possess a pure-strategy Nash equilibrium.

Thus, the most minor graph-theoretic extension of the D'Aspremont et al. (1979) two-firm line model with quadratic transportation costs is sufficient to lead to a non-existence result. The proof shows that no pure-strategy equilibrium exists if all firms are at equidistance from the junction and the longest edge is not inhabited by any of the firms. Consider Figure9. Intuitively,

\footnotetext{
${ }^{17}$ All these consumers are at equidistance from firm 1 and 2 , so this is an unshielded hinterland to both firm 1 and firm 2 .

${ }^{18}$ For $1.26<\alpha<5 / 3$, equilibria in locations exist but the locations of the equilibria are strictly interior.
} 

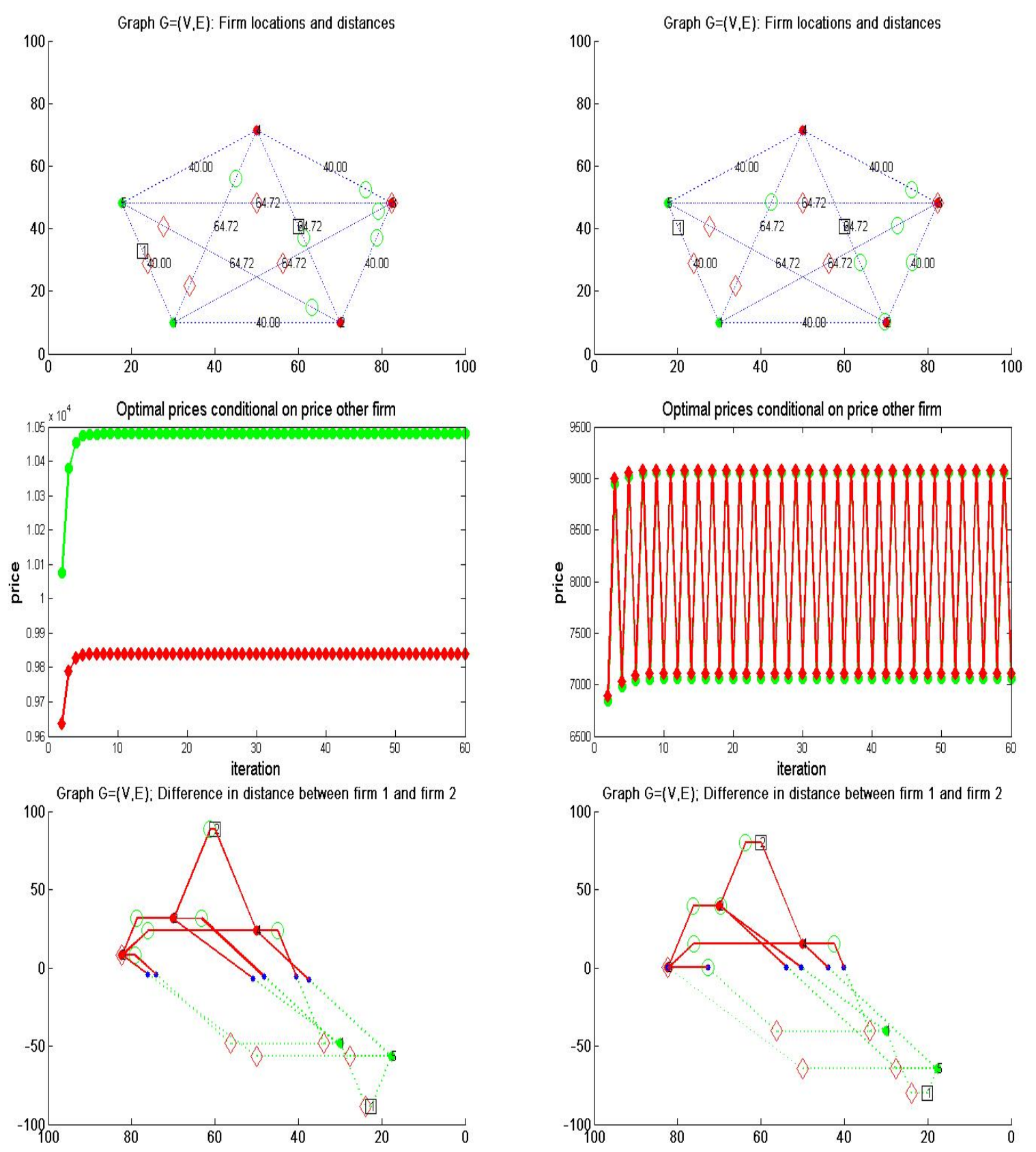

Figure 12: Examples of a complete graph with five nodes (pentagon). In the panels left, the distance between firm 1 and node 5 is 0.4 times the total length of edge $e_{15}$. A price equilibrium in pure strategies exists for this constellation $\left(p_{1}^{*} \approx 10479 ; p_{2}^{*} \approx 9840\right)$. In the right panels, the distance between firm 1 and node 5 is 0.2 times the total length of edge $e_{15}$. In this situation, no equilibrium exists, the reason being that the unshielded hinterland at edge $e_{14}$ has increased and has become the cause of an ongoing price war. 
the equidistance condition implies that all consumers along edge $e_{C D}$ will always flock to the same supplier. As a result, competition will be heavy, but once prices are low, each firm will find it in its interest to raise price to increase its profits. The result will probably hold for all $K_{1, q}$ graphs with $q>2$.

A brief comparison of this result with Varian's non-existence result (1980, Proposition 2) is appropriate. In Varian, the absence of symmetric price equilibria is due to the assumption of declining average cost curves and the fact that a slight price cut by one of the stores leads this store to capture all informed consumers. The behavior of these informed customers is akin to the flocking of consumers along edge $e_{C D}$ to the firm charging the lowest price including transportation cost. However, whereas in Varian's model each firm receives an equal share of uninformed consumers, firms in the current model may have asymmetric hinterlands and thus different incentives to cut price to attract the "informed customers". Other than in Varian's model, a noncooperative equilibrium in pure strategies may therefore exist unless the fraction of informed customers - i.e. the length of $e_{C D}$ - is sufficiently large for firms to start a price war. The proof essentially shows that in every $K_{1,3}$ graph one can position the firms such that this condition holds 19

I conjecture, but do not prove, that this result holds for all graph models of price competition involving two or more firms:

Conjecture 9 For every graph $G=(V, E)$ with a least one node having degree 3 or higher, there exists a configuration of firm locations for which the price competition game does not possess a pure-strategy Nash equilibrium.

The condition that at least of the graph's has to be of degree 3 or higher rules out the structures for which we know that they do have a pure-strategy equilibrium, such as the line and circle.

\subsection{Mixed-strategy price equilibria}

The unshielded hinterland causes demand discontinuities that lead to non-existence of equilibria in pure strategies. However, for the model with two firms, mixed-strategy price equilibria do exist because the profit functions $\pi_{i}\left(p_{1}, p_{2}\right)(i=1,2)$ are bounded and weakly lower semi-continuous

\footnotetext{
${ }^{19}$ In Economides (1986b), consumers are evenly distributed on a surface. He shows that demand and profit functions are continuous for fairly general distance functions including the Euclidean metric but not for the block metric. In our application, the distance between two points $x$ and $y$ is determined by the length of the shortest path between these two points. That is, we cannot use a different distance function to remove the "thickness" of consumers at the boundary to restore existence.
} 
in $p_{i}$ and $\sum_{i=1}^{2} \pi_{i}(\mathbf{p})$ is upper-semicontinuous (Dasgupta and Maskin, 1986a, Theorem 5). For the model with two firms, we therefore have the following positive result, which essentially extends Theorem 3 in Dasgupta and Maskin (1986b).

Theorem 10 The two-firm graph model of price competition has a mixed-strategy equilibrium for all configurations of firm locations.

This theorem does not use the assumption of quadratic transportation cost. This implies that the result extends to graph models where consumers face other forms of nonlinear or linear transportation cost.

\section{Summary and discussion}

This paper is a first contribution to the analysis of graph models of price competition. The algorithm introduced allows one to numerically evaluate firm-level demand and profits for all graphs where consumers are uniformly distributed along the edges and face quadratic transportation cost and where two firms compete in prices conditional on their location. One important phenomenon for this type of models is that spatial discontinuities in demand may occur. The most important result is that the existence result by D'Aspremont et al. (1979) for the $K_{1,2}$ graph does not extend to the $K_{1,3}$ graph, arguably the most straightforward extension of the original model.

I believe that the framework presented in this paper offers ample scope for future research. Besides proving or falsifying the conjecture on the non-existence of pure-strategy price equilibria for graphs, natural directions for further investigation include the analysis of markets with three or more firms, issues related to endogenous entry and markets where consumers face non-linear, but not necessarily quadratic transportation costs. Furthermore, whereas the present paper presents numerical evaluations for a number of specific graphs, it is worthwhile to investigate more systematically the relationship between graph characteristics, firm locations within the graph and pricing equilibria. One of the results in D'Aspremont et al. (1979) is that for the line model with linear transportation cost, pure-strategy equilibria exist if firms are far enough apart. Are there classes of graphs for which a similar result can be obtained?

Another avenue for research is the study of the relationship between graph characteristics, firm location and the occurrence and characteristics (length, amplitude, symmetry) of price cycles. Theoretical Edgeworth cycles, first described by Edgeworth (1925) and given a solid 
game-theoretic foundations by Maskin and Tirole (1988), are characterized by strongly asymmetric periods of price cuts followed by a rapid price increase. Theoretically, Edgeworth price cycles are most likely to occur in markets characterized by homogenous goods and extremely price-sensitive consumers. Consistent with this, one particular market in which asymmetric price cycles have been consistently found is the market for retail gasoline. Typically, these studies start with the observation of price cycles in a certain market, verify whether or not the cycles are asymmetric, and, conditional on finding asymmetries, look for the possible causes 20 Noel (2009) for example decomposes asymmetric price cycles into a part that can be explained by Edgeworth cycles and a part driven by other unknown sources. Less attention has been paid to why some firms are cycling and other are not. Exceptions are Noel (2007a) and De Roos and Katayama (2010) who use a Markov switching-regression model and allow for differences in the price cycles of major firms and independents. The location of a firm on a given road network relative the location of its competitors might be an important additional variable explaining the occurrence and shape of these price cycles.

\footnotetext{
${ }^{20}$ These empirical studies give evidence for price cycles in the US (Castanias and Johnson, 1993; Lewis (forthcoming); Lewis and Noel (forthcoming)), Canada (Noel, 2007a, 2007b; Eckert, 2003), Australia (Wang, 2009; De Roos and Katayama, 2010). Bachmeier and Griffin, 2003 do not uncover asymmetric cycles.
} 


\section{Appendix}

\section{Proof of Lemma 1}

Suppose there are two different change points $c_{\omega, \omega^{\prime}}^{i}$ and $\tilde{c}_{\omega, \omega^{\prime}}^{i}$ at line segment $e_{\omega, \omega^{\prime}} \backslash\left\{\omega, \omega^{\prime}\right\}$. Then there exist different shortest paths $P_{c_{\omega, \omega^{\prime}}^{i}}^{S_{1}} f_{i}$ and $P_{c_{\omega, \omega^{\prime}}^{i}, f_{i}}^{S_{2}}$, and $P_{\tilde{c}_{\omega, \omega^{\prime}}^{i}, f_{i}}^{S_{1}}$ and $P_{\tilde{c}_{\omega, \omega^{\prime}}^{i}}^{S_{2}}$, such that

$$
\begin{gathered}
d\left(P_{c_{\omega, \omega^{\prime}}^{i} f_{i}}^{S_{1}}\right)=d\left(P_{c_{\omega, \omega^{\prime}}^{i}, f_{i}}^{S_{2}}\right) \text { and } d\left(P_{\tilde{c}_{\omega, \omega^{\prime}}^{i}, f_{i}}^{S_{1}}\right)=d\left(P_{\tilde{c}_{\omega, \omega^{\prime}}^{i}, f_{i}}^{S_{2}}\right) \\
\omega=V^{1}\left(P_{c_{\omega, \omega^{\prime}}^{i}, f_{i}}^{S_{1}}, V\right) \neq V^{1}\left(P_{c_{\omega, \omega^{\prime}}^{i}, f_{i}}^{S_{2}}, V\right)=\omega^{\prime}
\end{gathered}
$$

and

$$
\omega=V^{1}\left(P_{\tilde{c}_{\omega, \omega^{\prime}}^{i}}^{S_{1}}, f_{i}, V\right) \neq V^{1}\left(P_{\tilde{c}_{\omega, \omega^{\prime}}^{i}, f_{i}}^{S_{2}}, V\right)=\omega^{\prime} .
$$

Since the change points are not identical, one of them, say $c_{\omega, \omega^{\prime}}^{i}$, is closer to $\omega$ and $\tilde{c}_{\omega, \omega^{\prime}}^{i}$ is closer to $\omega^{\prime}$. This implies that $d\left(P_{c_{\omega, \omega^{\prime}}^{i} f_{i}}^{S_{1}}\right)<d\left(P_{\tilde{c}_{\omega, \omega^{\prime}}^{i}, f_{i}}^{S_{1}}\right)$ and $d\left(P_{c_{\omega, \omega^{\prime}}^{i}, f_{i}}^{S_{2}}\right)>d\left(P_{\tilde{c}_{\omega, \omega^{\prime}}^{i}, f_{i}}^{S_{2}}\right)$. This contradicts A.1) so $c_{\omega, \omega^{\prime}}^{i}$ and $\tilde{c}_{\omega, \omega^{\prime}}^{i}$ cannot both be change points ${ }^{21}$

\section{Proof of Lemma 2}

The shortest distance between two points is the length of the line connecting the two points. If $f_{i} \in e_{\omega, \omega^{\prime}}$ the shortest path distance from each point $x \in e_{\omega, \omega^{\prime}}$ to $f_{i}$ is equal to the length of the line segment $e_{x, f_{i}}, d\left(e_{x, f_{i}}\right)$. For these $x$ 's, there cannot exist two distinct shortest paths such that $d\left(P_{x, f_{i}}^{S_{1}}\right)=d\left(P_{x, f_{i}}^{S_{2}}\right)=d\left(e_{x, f_{i}}\right)$. In other words, none of the points $x \in e_{\omega, \omega^{\prime}}$ can be a change point with respect to $f_{i}$.

\section{Proof of Lemma 3}

First I prove the necessity of the conditions for interior change points. From Lemma 2 we know that if $e_{\omega, \omega^{\prime}}$ contains a change point w.r.t. $f_{i}$, it cannot contain $f_{i}$ itself. Suppose that $c$ is a change point for $f_{i}$ at the interior of $e_{\omega, \omega^{\prime}}$ and that the distance from $c$ to $\omega$ is $\epsilon>0$. From the definition of change points, it follows that there exist shortest paths $P_{c, f_{i}}^{S_{1}}$ and $P_{c, f_{i}}^{S_{2}}$ such that

$$
d\left(P_{c, f_{i}}^{S_{1}}\right)=d\left(P_{c, f_{i}}^{S_{2}}\right), V^{1}\left(P_{c, f_{i}}^{S_{1}}, V\right)=\omega \text { and } V^{1}\left(P_{c, f_{i}}^{S_{2}}, V\right)=\omega^{\prime}
$$

Because

$$
d\left(P_{c, f_{i}}^{S_{1}}\right)=d\left(P_{c, f_{i}}^{S_{2}}\right) \Leftrightarrow d\left(P_{\omega, f_{i}}^{S}\right)+\epsilon=d\left(P_{\omega^{\prime}, f_{i}}^{S}\right)+\left[d\left(e_{\omega, \omega^{\prime}}\right)-\epsilon\right]
$$

\footnotetext{
${ }^{21}$ Note that the change point $c_{\omega}^{i}$ at the endpoint $\omega$ in Figure 2 would not satisfy equation A.2.
} 
it follows that

$$
\left|d\left(P_{\omega, f_{i}}^{S}\right)-d\left(P_{\omega^{\prime}, f_{i}}^{S}\right)\right|=\left|d\left(e_{\omega, \omega^{\prime}}\right)-2 \epsilon\right|<d\left(e_{\omega, \omega^{\prime}}\right)
$$

Sufficiency follows from noting that when $e_{\omega, \omega^{\prime}}$ contains no change point and $f_{i} \notin e_{\omega, \omega^{\prime}}, d\left(P_{\omega^{\prime}, f_{i}}^{S}\right)=$ $d\left(P_{\omega, f_{i}}^{S}\right)+d\left(e_{\omega, \omega^{\prime}}\right)$.

Next consider the presence of a change points on one of the endpoints of $e_{\omega, \omega^{\prime}}$, say $\omega$. For $\omega$ being a change point, it follows that there exist two shortest paths such that

$$
V^{1}\left(P_{\omega, f_{i}}^{S_{1}}, V\right) \neq V^{1}\left(P_{\omega, f_{i}}^{S_{2}}, V\right)
$$

Take $v=V^{1}\left(P_{\omega, f_{i}}^{S_{1}}, V\right)$ and $v^{\prime}=V^{1}\left(P_{\omega, f_{i}}^{S_{2}}, V\right)$. Because $\omega$ is a change point,

$$
d\left(P_{\omega, f_{i}}^{S_{1}}\right)=d\left(P_{\omega, f_{i}}^{S_{2}}\right)=d\left(P_{\omega, f_{i}}^{S}\right)
$$

which implies

$$
d\left(P_{\omega, f_{i}}^{S_{1}}\right)=d\left(e_{\omega, v}\right)+d\left(P_{v, f_{i}}^{S}\right)=d\left(e_{\omega, v^{\prime}}\right)+d\left(P_{v^{\prime}, f_{i}}^{S}\right),
$$

and thus the conditions in Lemma 3 are satisfied. This proves the necessity of the conditions.

To prove sufficiency suppose that $\omega$ is an endpoint of $e_{\omega, \omega^{\prime}}$ but not a change point. According to $b$ ), we know that there exist two vertices $v$ and $v^{\prime}$ adjacent to $\omega$ and that

$$
d\left(P_{\omega, f_{i}}^{S}\right)=d\left(e_{\omega, v}\right)+d\left(P_{v, f_{i}}^{S}\right)=d\left(e_{\omega, v^{\prime}}\right)+d\left(P_{v^{\prime}, f_{i}}^{S}\right) .
$$

If $\omega$ is not a change point, it must hold that for one of these vertices, say $v$, there exists a point $\omega^{*}$ with

$$
\omega^{*}=\omega+\lambda(v-\omega), \text { with } \lambda>0,
$$

such that

$$
d\left(P_{\omega^{*}, f_{i}}^{S}\right)>d\left(P_{\omega, f_{i}}^{S}\right)
$$

However, since $\omega^{*}$ is closer to $v$ than $\omega$, it must also hold that $d\left(e_{\omega^{*}, v}\right)<d\left(e_{\omega, v}\right)$. But this and equation A.4 together imply that

$$
d\left(P_{\omega^{*}, f_{i}}^{S}\right)=d\left(e_{\omega}^{*}, v\right)+d\left(P_{v, f_{i}}^{S}\right)<d\left(P_{\omega, f_{i}}^{S}\right)
$$

and we arrive at a contradiction. Thus $\omega$ must be a change point. 


\section{Proof of Proposition 4}

From $\omega \in S_{f_{i}}$ it follows that at point $\omega$, the utility difference between buying from firm $i$ and buying from firm $j \neq i$ is nonnegative:

$$
\Delta U \equiv U\left(\omega, d\left(\omega, f_{i}\right), \mathbf{p}\right)-U\left(\omega, d\left(\omega, f_{j}\right), \mathbf{p}\right)=c\left[d\left(\omega, f_{j}\right)^{\alpha}-d\left(\omega, f_{i}\right)^{\alpha}\right]+\left(p_{j}-p_{i}\right) \geq 0
$$

with the difference equal to zero if and only if $\omega \in S_{f_{j}}^{e}$. Write $d\left(\omega, f_{j}\right)=d\left(\omega, f_{i}\right)+z$ with $z>0$ if and only if $\omega \in C_{f_{i}}$ and $\omega \notin C_{f_{j}} ; z<0$ if and only if $\omega \notin C_{f_{i}}$ and $\omega \in C_{f_{j}}$, and $z=0$ if and only if $\omega \in C_{f_{i}}$ and $\omega \in C_{f_{j}}$. By definition, for the location $y \in e_{\omega, \omega^{\prime}}$ of a marginal consumer, it has to hold that

$$
\Delta U=c\left[d\left(y, f_{2}\right)^{\alpha}-d\left(y, f_{1}\right)^{\alpha}\right]+\left(p_{2}-p_{1}\right)=0 .
$$

For ease of exposition, we denote by $x \geq 0$ the distance between the points $\omega$ and $y, x \equiv d(\omega, y)$.
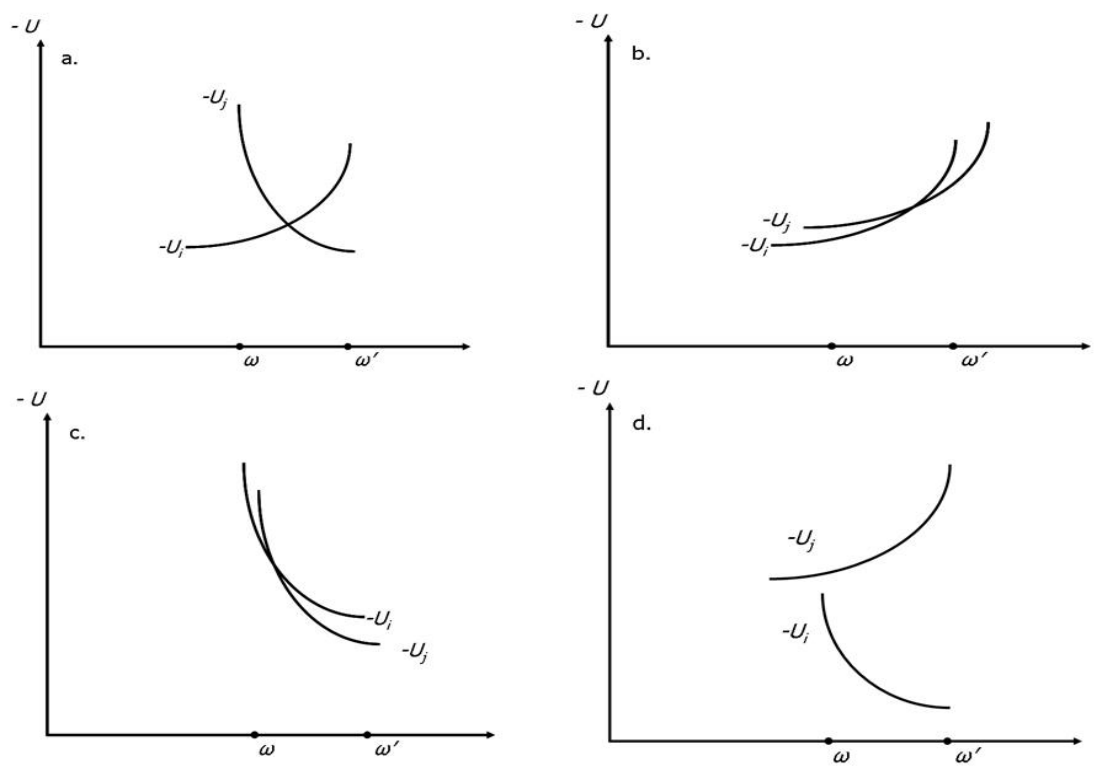

Figure 13: Examples of changes in utility when moving along $e_{\omega, \omega^{\prime}}$

Given that no change points are present at the interior of $e_{\omega, \omega^{\prime}}$, we have one of the following situations:

a. $d_{\omega, \omega^{\prime}}^{j}=-1$ and $d_{\omega, \omega^{\prime}}^{i}=+1$

b. $d_{\omega, \omega^{\prime}}^{j}=+1$ and $d_{\omega, \omega^{\prime}}^{i}=+1$;

c. $d_{\omega, \omega^{\prime}}^{j}=-1$ and $d_{\omega, \omega^{\prime}}^{i}=-1$, or 
d. $d_{\omega, \omega^{\prime}}^{j}=+1$ and $d_{\omega, \omega^{\prime}}^{i}=-1$.

Figure 5 shows examples of each of the four cases.

Note that $\partial d\left(y, f_{i}\right) / \partial x=d_{\omega, \omega^{\prime}}^{i}$ and $\partial d\left(y, f_{j}\right) / \partial x=d_{\omega, \omega^{\prime}}^{j}$ such that the derivative of $\Delta U$ with respect to $x$ can be written as

$$
\begin{aligned}
\frac{\partial \Delta U}{\partial x} & =\alpha c\left[d\left(y, f_{j}\right)^{\alpha-1} \frac{\partial d\left(y, f_{j}\right)}{\partial x}-d\left(y, f_{i}\right)^{\alpha-1} \frac{\partial d\left(y, f_{i}\right)}{\partial x}\right] \\
& =\alpha c\left[d\left(y, f_{j}\right)^{\alpha-1} d_{\omega, \omega^{\prime}}^{j}-d\left(y, f_{i}\right)^{\alpha-1} d_{\omega, \omega^{\prime}}^{i}\right] .
\end{aligned}
$$

Furthermore,

$$
d\left(y, f_{j}\right)=d_{\omega, \omega^{\prime}}^{j} \cdot x+d\left(\omega, f_{j}\right)=d_{\omega, \omega^{\prime}}^{j} \cdot x+d\left(\omega, f_{i}\right)+z
$$

and

$$
d\left(y, f_{i}\right)=d_{\omega, \omega^{\prime}}^{i} \cdot x+d\left(\omega, f_{i}\right)
$$

such that

$$
d\left(y, f_{j}\right)-d\left(y, f_{i}\right)=\left(d_{\omega, \omega^{\prime}}^{j}-d_{\omega, \omega^{\prime}}^{i}\right) x+z .
$$

For situations a to $\mathbf{d}$, we thus have

a. $d\left(y, f_{j}\right)-d\left(y, f_{i}\right)=-2 x+z$

b and c. $d\left(y, f_{j}\right)-d\left(y, f_{i}\right)=z$;

d. $d\left(y, f_{j}\right)-d\left(y, f_{i}\right)=2 x+z$.

Now first consider the case for which $\omega \notin S_{f_{j}}$, that is, the inequality in equation A.5 is strict. This implies that there can only be a marginal consumer $y \in e_{\omega, \omega^{\prime}}$ if $\partial \Delta U / \partial x<0$. From equation A.7 it readily follows that when situation a obtains, $\partial \Delta U / \partial x$ is positive for all points $y \in e_{\omega, \omega^{\prime}}$. For situation $\mathbf{b}(\mathbf{c}), \partial \Delta U / \partial x<0$ only if $z<0(z>0)$ and for situation $\mathbf{d}, \partial \Delta U / \partial x<0$ does not impose conditions on $z$. Since $z<0(z>0)$ if and only if $\left.\omega \notin C_{f_{i}} \wedge \omega \in C_{f_{j}}\right)\left(\omega \in C_{f_{i}} \wedge \omega \notin C_{f_{j}}\right)$ ).

In case $\omega \in S_{f_{j}}$, the point of departure is itself the location of a marginal consumer and points on the interior of $e_{\omega, \omega^{\prime}}$ are locations of marginal consumers if and only if $\partial \Delta U / \partial x$ for all $x \in\left[0, d\left(\omega, \omega^{\prime}\right)\right]$. That is, if and only if

$$
d_{\omega, \omega^{\prime}}^{i}=d_{\omega, \omega^{\prime}}^{j} \wedge d\left(\omega, f_{i}\right)=d\left(\omega, f_{j}\right)
$$

that is, the distance to either firm should be increasing or decreasing when moving along $e_{\omega, \omega^{\prime}}$ from $\omega$ to $\omega^{\prime}$ and point $\omega$ is at equidistance from firm $i$ and firm $j: \omega \in C_{f_{i}} \wedge \omega \in C_{f_{j}}$. This may 
happen only in situations $\mathbf{b}$ and $\mathbf{c}$. In situation $\mathbf{a}, e_{\omega, \omega^{\prime}} \backslash\{\omega\} \notin S_{f_{i}}$ and $e_{\omega, \omega^{\prime}} \in S_{f_{j}}$; in situation $\mathbf{d}, e_{\omega, \omega^{\prime}} \in S_{f_{i}}$ and $e_{\omega, \omega^{\prime}} \backslash\{\omega\} \notin S_{f_{j}}$. This completes the proof.

\section{Proof of Corollary 5}

Suppose $\omega \in S_{f_{i}}, \omega \in C_{f_{i}}$ and $e_{\omega, \omega^{\prime}} \backslash\{\omega\}$ contains a change point $c$ with respect to firm $j$. Then $d_{\omega, c}^{j}=+1$ on the line segment $e_{\omega, c} \subseteq e_{\omega, \omega^{\prime}}$. None of the conditions in Proposition 4 is satisfied and thus $e_{\omega, \omega^{\prime}}$ does for inhabit a marginal consumer if $\omega \notin S_{f_{j}}$; according to Proposition 4 , for $\omega \in S_{f_{i}}$, all consumers at $e_{\omega, \omega^{\prime}} \backslash\{\omega\}$ are indifferent between buying from either firm if and only if $d_{\omega, \omega^{\prime}}^{i}=d_{\omega, \omega^{\prime}}^{j}=+1$ and $\omega \in C_{f_{i}}$ and $\omega \in C_{f_{j}}$.

\section{Proof of Theorem 8}

Consider $K_{1,3}$ graph as shown in Figure 95, with $d\left(A, f_{1}\right)=\eta, d\left(f_{1}, D\right)=\delta, d\left(D, f_{2}\right)=\epsilon$, $d\left(f_{2}, B\right)=\theta$ and $d\left(e_{C D}\right) \equiv \xi . d\left(e_{A D}\right) \equiv \eta+\delta$ and $d\left(e_{B D}\right) \equiv \epsilon+\theta$. The proof consists of showing that for any value of $d_{A D}, d_{B D}$ and $d\left(e_{C D}\right)$, there exists at least one configuration of firm locations for which no equilibrium in pure strategies exist.

Assume without loss of generality that $e_{C D}$ is at least as long as the two other edges, i.e. $\xi \geq d\left(e_{A D}\right)$ and $\xi \geq d\left(e_{B D}\right)$. Locate firm 1 at edge $e_{A D}$ and firm 2 at edge $e_{B D}$ each firm at one of the other two edges. Furthermore, assume that $\epsilon \geq \delta>0$, that is: firm 1 is at least as close to node $D$ as firm 2 .

First consider the decision problem for consumers located at vertices $A-D$ :

- Consumers at vertex $A$ are indifferent if $p_{1}=p_{2}+c(\delta+\epsilon)^{2}+2 c \eta(\delta+\epsilon)$;

- consumers at vertex $B$ are indifferent if $p_{1}=p_{2}-c(\delta+\epsilon)^{2}-2 c \theta(\delta+\epsilon)$;

- consumers at vertex $C$ are indifferent if $p_{1}=p_{2}+c\left(\epsilon^{2}-\delta^{2}\right)+2 c \xi(\epsilon-\delta)=p_{2}+z_{1}+z_{2}$;

- consumers at vertex $D$ are indifferent if $p_{1}=p_{2}+c\left(\epsilon^{2}-\delta^{2}\right)=p_{2}+z_{1}$,

with $z_{1} \equiv c\left(\epsilon^{2}-\delta^{2}\right)$ and $z_{2} \equiv 2 c \xi(\epsilon-\delta)$. Note that $z_{1}, z_{2} \geq 0$ iff. $\epsilon \geq \delta$.

This implies that firm 1 receives zero demand if

$$
p_{1}>p_{2}+c \max \left\{(\delta+\epsilon)^{2}+2 \eta(\delta+\epsilon), \epsilon^{2}-\delta^{2}+2 \xi(\epsilon-\delta)\right\}
$$

and firm 2 receives zero demand if

$$
p_{1}<p_{2}-c(\delta+\epsilon)^{2}-2 c \theta(\delta+\epsilon)
$$


see Figure 14 for a graphical representation.

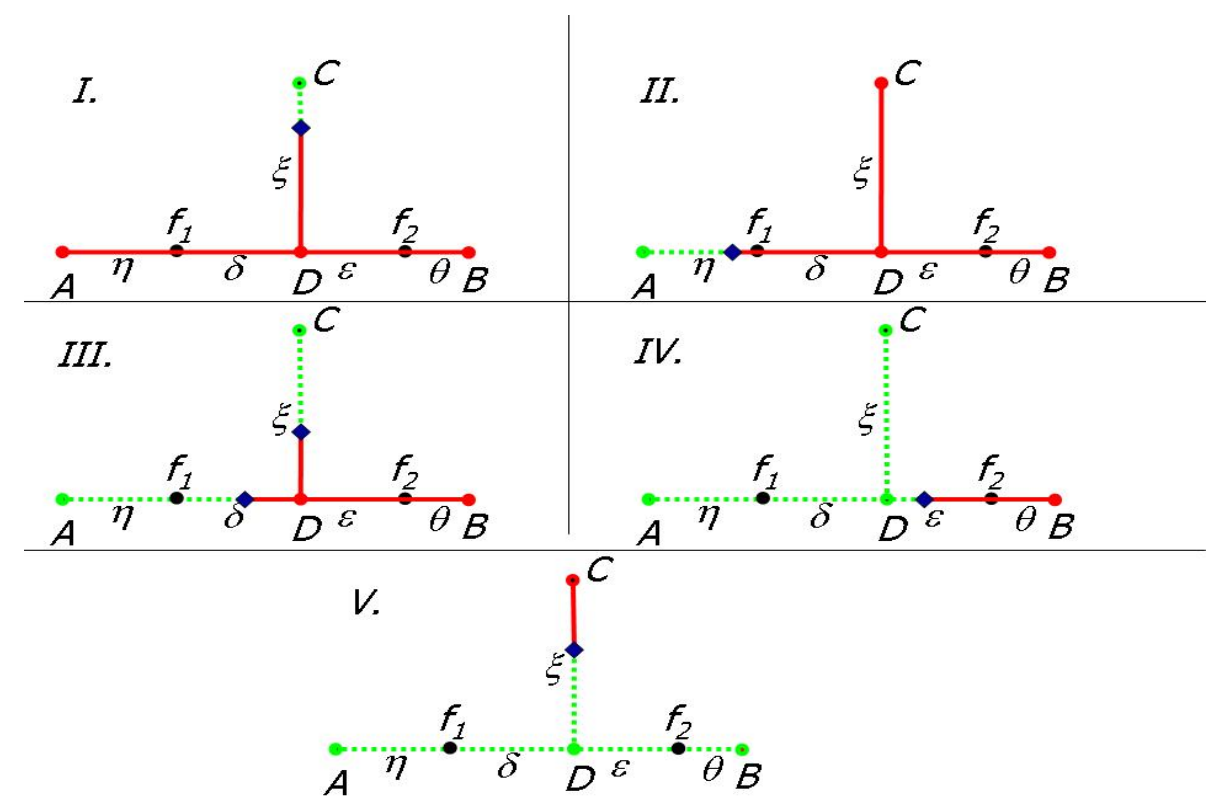

Figure 14: Proof Theorem 8. Market division into demand for firm 1 (dotted green lines) and demand for firm 2 (solid red lines). The blue diamonds represent marginal consumers.

Figure 14 shows that demand for firm 1 falls to zero when consumers at vertex $C$ (panel $I$ ) or vertex $A$ (panel $I I$ ) become indifferent between buying from either firm. The first (second) panel applies when $\xi$ is relatively large (small) compared to $\eta$. Note that demand for firm 2 falls to zero once consumers at vertex $B$ become indifferent (panel $I V$ ); a situation as depicted in panel $V$ - with consumers at vertex $B$ buying from firm 1 but firm 2 still receiving positive demand from $e_{C D}$ - cannot occur because we assume that $\epsilon \geq \delta$.

This implies that any equilibrium $\left(p_{1}^{*}, p_{2}^{*}\right)$ must satisfy the condition

$$
\left|p_{1}^{*}-p_{2}^{*}\right| \leq c \max \left\{(\delta+\epsilon)^{2}+2(\delta+\epsilon) \max (\eta, \theta), \epsilon^{2}-\delta^{2}+2 \xi(\epsilon-\delta)\right\},
$$

because otherwise, one of the two firms may gain from decreasing its price to the delivered price of the other.

In the remaining part of the proof, I assume that $\left(\epsilon^{2}-\delta^{2}\right)+2 \xi(\epsilon-\delta) \leq(\delta+\epsilon)^{2}+2 \eta(\delta+\epsilon)$, i.e. $\xi \leq \xi^{*} \equiv(\delta+\epsilon)(\delta+\eta) /(\delta-\epsilon)$. This condition - which is always satisfied for $\epsilon$ close enough to $\delta$-guarantees that a situation as in panel $I$ of Figure 14 will not occur. 
In this case, the profit functions of firm 1 and 2 are given by:

$$
\pi_{1}\left(p_{1}, p_{2}\right)=\left\{\begin{array}{lll}
(\xi+\eta+\delta+\epsilon+\theta) p_{1} & \text { if } & p_{1} \leq p_{2}-c(\delta+\epsilon)(\delta+\epsilon+2 \theta) \\
\left(\xi+\eta+\frac{p_{2}-p_{1}}{2 c(\delta+\epsilon)}+\frac{\delta+\epsilon}{2}\right) p_{1} & \text { if } & p_{2}-c(\delta+\epsilon)(\delta+\epsilon+2 \theta)<p_{1} \leq p_{2}+z_{1} \\
\left(\xi+\eta+\frac{\epsilon\left(p_{2}-p_{1}\right)}{c\left(\epsilon^{2}-\delta^{2}\right)}+\delta+\epsilon\right) p_{1} & \text { if } & p_{2}+z_{1}<p_{1} \leq p_{2}+z_{1}+z_{2} \\
\left.\eta+\frac{p_{2}-p_{1}}{2 c(\delta+\epsilon)}+\frac{\delta+\epsilon}{2}\right) p_{1} & \text { if } & p_{2}+z_{1}+z_{2}<p_{1} \leq p_{2}+c(\delta+\epsilon)(\delta+\epsilon+2 \eta) \\
0 & \text { if } & p_{1}>p_{2}+c(\delta+\epsilon)(\delta+\epsilon+2 \eta)
\end{array}\right.
$$

and

$$
\pi_{2}\left(p_{1}, p_{2}\right)=\left\{\begin{array}{lll}
1 & \text { if } & p_{2}<p_{1}-c(\delta+\epsilon)(\delta+\epsilon+2 \eta) \\
\left(\xi+\theta+\frac{p_{1}-p_{2}}{2 c(\delta+\epsilon)}+\frac{\delta+\epsilon}{2}\right) p_{2} & \text { if } & p_{1}-c(\delta+\epsilon)(\delta+\epsilon+2 \eta) \leq p_{2}<p_{1}-z_{1}-z_{2} \\
\left.\theta+\frac{\epsilon\left(p_{1}-p_{2}\right)}{c\left(\epsilon^{2}-\delta^{2}\right)}\right) p_{2} & \text { if } & p_{1}-z_{1}-z_{2} \leq p_{2}<p_{1}-z_{1} \\
\left.\theta+\frac{p_{1}-p_{2}}{2 c(\delta+\epsilon)}+\frac{\delta+\epsilon}{2}\right) p_{2} & \text { if } & p_{1}-z_{1} \leq p_{2}<p_{1}+c(\delta+\epsilon)((\delta+\epsilon)+2 \theta) \\
0 & \text { if } & p_{2} \geq p_{1}+c(\delta+\epsilon)(\delta+\epsilon+2 \theta)
\end{array}\right.
$$

Suppose that $\left(p_{1}^{*}, p_{2}^{*}\right)$ is an equilibrium and that $p_{2}^{*}-c(\delta+\epsilon)(\delta+\epsilon+2 \theta)<p_{1}^{*} \leq p_{2}^{*}+z_{1}$. Taking first order conditions for the relevant part of the profit functions gives us

$$
\begin{aligned}
& p_{1}^{*}=\frac{4}{3} c(\delta+\epsilon)\left[\xi+\eta+\frac{1}{2} \theta\right]+c(\delta+\epsilon)^{2} \\
& p_{2}^{*}=\frac{4}{3} c(\delta+\epsilon)\left[\theta+\frac{1}{2}(\xi+\eta)\right]+c(\delta+\epsilon)^{2}
\end{aligned}
$$

This set of prices is within the given range if

$$
p_{1}^{*}-p_{2}^{*} \leq c\left(\epsilon^{2}-\delta^{2}\right) \Leftrightarrow 2(\xi+\eta-\theta) \leq \epsilon-\delta \Leftrightarrow \xi+d_{A D}-d_{B D} \leq(\epsilon-\delta) / 2 .
$$

Such an equilibrium with firm 1 charging low prices, which corresponds to panel $I V$ in Figure 14, thus only occurs when $\xi$ or $\eta$ are relatively small or $\theta$ is relatively large. Intuitively, these are situations for which firm 1 is very competitive because its shielded hinterland $(\eta)$ is small, firm 2 has a large shielded hinterland $(\theta)$ of itself and/or the unshielded hinterland $(\xi)$ is too small for firm 2 to warrant price cuts.

Now suppose that $\left(p_{1}^{*}, p_{2}^{*}\right)$ is an equilibrium but that $p_{2}^{*}+z_{1}<p_{1}^{*} \leq p_{2}^{*}+z_{1}+z_{2}$, a situation as in panel III. Again taking first order conditions for the relevant part of the profit functions gives us

$$
\begin{aligned}
& p_{1}^{*}=\frac{2 c}{3 \epsilon}\left(\xi+d_{A D}+\frac{1}{2}\left(d_{B D}+\epsilon\right)\right)\left(\epsilon^{2}-\delta^{2}\right) \\
& p_{2}^{*}=\frac{2 c}{3 \epsilon}\left(\frac{1}{2}\left(\xi+d_{A D}\right)+d_{B D}-\frac{\epsilon}{2}\right)\left(\epsilon^{2}-\delta^{2}\right)
\end{aligned}
$$


This set of prices is within the given range if

$$
\xi+d_{A D}-d_{B D} \leq \frac{6 \xi \epsilon}{\delta+\epsilon}+\epsilon \quad \text { and } \quad \xi+d_{A D}-d_{B D}>3 \epsilon
$$

Intuitively, the likelihood of observing an equilibrium situation as in panel $I I I$, with demand at $e_{B C}$ split between firm 1 and 2 is increasing with $\xi$.

Finally suppose that $\left(p_{1}^{*}, p_{2}^{*}\right)$ is an equilibrium with $p_{2}+z_{1}+z_{2}<p_{1} \leq p_{2}+c(\delta+\epsilon)(\delta+\epsilon+2 \eta)$, a situation depicted in panel $I I$. Taking first order conditions for the relevant part of the profit functions now yields

$$
\begin{aligned}
& p_{1}^{*}=\frac{4}{3} c(\delta+\epsilon)\left[\eta+\frac{1}{2}(\xi+\theta)\right]+c(\delta+\epsilon)^{2} \\
& p_{2}^{*}=\frac{4}{3} c(\delta+\epsilon)\left[\xi+\theta+\frac{1}{2} \eta\right]+c(\delta+\epsilon)^{2}
\end{aligned}
$$

For this set of prices, $p_{1}^{*}>p_{2}^{*}+z_{1}+z_{2}$ if and only if

$$
\xi+d_{A D}-d_{B D}>2 \xi+(\epsilon-\delta)+\frac{3 \xi(\epsilon-\delta)}{\epsilon+\delta}
$$

Note that $0<\xi+d_{A D}-d_{B D}<2 \xi$ because we have assumed that $\xi \geq d_{A D}$ and $\xi \geq d_{B D}$. Now take $\epsilon=\delta>0$, that is, both firms are located at equidistance from vertex $D$. From the above it is clear that for this configuration of firm locations, no price equilibrium in pure strategies exists: the pairs $\left(p_{1}^{*}, p_{2}^{*}\right)$ in $\mathrm{A} .8$ ) and $\mathrm{A} .12$ are not an equilibrium because the conditions A.9 and A.13 are not satisfied when $\epsilon=\delta$ and the pair $\left(p_{1}^{*}, p_{2}^{*}\right)$ in A.10 is not an equilibrium because for $\epsilon=\delta, p_{1}^{*}=p_{2}^{*}=0$ and each firm can strictly increase profits by slightly increasing its price. This completes the proof ${ }^{22}$

\footnotetext{
${ }^{22}$ As an example of a situation for which an equilibrium does exist, take $\xi=24, \eta=5, \delta=1, \epsilon=2$ and $\theta=6$, such that $d_{A D=6}$ and $d_{B D}=8$. In this case, condition A.11 is satisfied, and A.10 gives $p_{1}^{*}=35$, and $p_{2}^{*}=22$ with corresponding profits $\pi_{1}\left(p_{1}^{*}, p_{2}^{*}\right)=1014$ and $\pi_{2}\left(p_{1}^{*}, p_{2}^{*}\right)=384$.
} 


\section{References}

Bachmeier, Lance J. and James M Griffin, "New Evidence on Asymmetric Gasoline Price Responses," Review of Economics and Statistics, August 2003, 85 (3), 772-776.

Bertsekas, Dimitri P., Linear Network Optimization: Algorithms and Codes, Cambridge, Massachusetts: MIT Press, 1991.

Bloch, Francis and Nicolas Quérou, "Pricing in Networks," working paper no. 2008-31, Ecole Polytechnique 2009.

Bollobás, Béla, Modern Graph Theory Graduate Texts in Mathematics, New York: Springer, 1998.

Bollobás, Belá and Nicolas Stern, "The Optimal Structure of Market Areas," Journal of Economic Theory, 1972, 4, 174-179.

Castanias, Rick and Herb Johnson, "Gas Wars: Retail Gasoline Price Fluctuations," Review of Economics and Statistics, February 1993, 75 (1), 171-174.

Claycombe, Richard J. and Tamara E. Mahan, "Spatial Aspects of Retail Market Structure: Beef Pricing Revisited," International Journal of Industrial Organization, June 1991, 11, 283-291.

Dasgupta, Partha and Eric Maskin, "The Existence of Equilibrium in Discontinuous Economic Games, I: Theory," The Review of Economic Studies, January 1986a, 53 (1), 1-26.

and __ , "The Existence of Equilibbrium in Discontinous Economic Games, II: Applications," Review of Economic Studies, January 1986b, 53 (1), 27-41.

D'Aspremont, C., J. Jaskold Gabszewicz, and J.-F. Thisse, "On Hotelling's "Stability in Competition", Econometrica, September 1979, 47 (5), 1145-1150.

de Roos, Nicolas and Hajime Katayama, "Retail Petrol Price Cycles in Western Australia," mimeo, University of Sydney 2010.

Eaton, B. Curtis and Richard G. Lipsey, "The Non-Uniqueness of Equilibrium in the Loschian Location Model," American Economic Review, March 1976, 66 (1), 77-93.

Eckert, Andrew, "Retail Price Cycles and the Presence of Small Firms," International Journal of Industrial Organization, February 2003, 21 (2), 151-170.

Economides, Nicolas, "Minimal and Maximal Product Differentiation in Hotelling's Duopoly," Economics Letters, 1986a, 21, 67-71.

, "Nash equilibrium in duopoly with products defined by two characteristics," RAND Journal of Economics, Autumn 1986b, 17 (3), 431-439.

Edgeworth, Francis, "The Pure Theory of Monopoly," in "Papers Relating to Political Economy," Vol. 1, London: MacMillan, 1925, pp. 111-142.

Greenhut, M. L., M. J. Hwang, and H. Ohta, "An Empirical Evaluation of the Equilibrium Size and Shape of Market Areas," International Economic Review, February 1976, 17 (1), 172-190.

Holahan, William L. and Richard E. Schuler, "The Welfare Effects of Market Shapes in the Loschian Location Model: Squares Vs. Hexagons," American Economic Review, September 1981, 71 (4), 738-746.

Hotelling, Harold, "Stability in Competition," Economic Journal, March 1929, 39 (153), 41-57.

Houde, Jean-François, "Spatial Differentiation and Vertical Mergers in Retail Markets for Gasoline," American Economic Review, forthcoming.

Irmen, Andreas and Jacques-François Thisse, "Competition in Multi-Characteristics Spaces: Hotelling Was Almost Right," Journal of Economic Theory, 1998, 78, 76-102.

Lewis, Matthew S., "Asymmetric Price Adjustment and Consumer Search: An Examination of the Retail Gasoline Market," Journal of Economics and Management Strategy, forthcoming 2010.

and Michael D. Noel, "The Speed of Gasoline Price Response in Markets with and Without Edgeworth Cycles," Review of Economics and Statistics, forthcoming 2010.

Lösch, Auguste, The Economics of Location, New Haven, Connecticut: Yale University Press, 1954.

Martin, Stephen, Advanced Industrial Economics, 2nd ed., Malden, Massachusetts, USA: Blackwell, 2002.

Maskin, Eric and Jean Tirole, "A Theory of Dynamic Oligopoly, II: Price Competition, Kinked Demand Curves, and Edgeworth Cycles," Econometrica, May 1988, 56 (3), 571-599. 
Mills, Edwin S. and Michael R. Lav, "A Model of Market Areas with Free Entry," The Journal of Political Economy, June 1964, 72 (3), 278-288.

Noel, Michael D., "Edgeworth Price Cycles: Evidence from the Toronto Retail Gasoline Market," Journal of Industrial Economics, March 2007a, LV (1), 69-92.

, "Edgeworth Price Cycles, Cost-Based Pricing and Sticky Pricing in Retail Gasoline Markets," Review of Economics and Statistics, May 2007b, 89 (2), 224-234.

, "Do Retail Gasoline Prices Respond Asymmetrically to Cost Shocks? The Influence of Edgeworth Cycles," RAND Journal of Economics, Autumn 2009, 40 (3), 582-595.

Osborne, Martin J. and Carolyn Pitchik, "Equilibrium in Hotelling's Model of Spatial Competition," Econometrica, July 1987, 55 (4), 911-922.

Picone, Gabriel A., David B. Ridley, and Paul A. Zandbergen, "Distance Decreases with Differentiation: Strategic Agglomeration by Retailers," International Journal of Industrial Organization, 2009, 27, $463-473$.

Raith, Michael, "Spatial Retail Markets with Commuting Consumers," International Journal of Industrial Organization, June 1996, 14 (4), 447-463.

Salop, Steven C., "Monopolistic Competition with Outside Goods," Bell Journal of Economics, Spring 1979, 10 (1), 141-156.

Varian, Hal R., "A Model of Sales," American Economic Review, September 1980, 70 (4), 651-659.

Von Ungern-Sternberg, Thomas, "Monopolistic Competition on the Pyramid," Journal of Industrial Economics, June 1991, 39 (4), 355-368.

Wang, Zhongmin, "(Mixed) Strategy in Oligopoly Pricing: Evidence from Gasoline Price Cycles Before and under a Timing Regulation," Journal of Political Economy, December 2009, 117 (6), 987-1030. 